\title{
BROADBAND OBSERVATIONS OF HIGH REDSHIFT BLAZARS
}

\author{
Vaidehi S. Paliya ${ }^{1,3}$, M. L. Parker ${ }^{2}$, A. C. Fabian ${ }^{2}$, and C. S. Stalin ${ }^{3}$ \\ ${ }^{1}$ Department of Physics and Astronomy, Clemson University, Kinard Lab of Physics, Clemson, SC 29634-0978, USA; vpaliya@g.clemson.edu \\ 2 Institute of Astronomy, Madingley Road, Cambridge CB3 OHA, UK \\ ${ }^{3}$ Indian Institute of Astrophysics, Block II, Koramangala, Bangalore-560034, India \\ Received 2016 April 12; revised 2016 April 25; accepted 2016 April 28; published 2016 June 29
}

\begin{abstract}
We present a multi-wavelength study of four high redshift blazars, S5 0014+81 $(z=3.37)$, CGRaBS J0225+1846 $(z=2.69)$, BZQ J1430+4205 $(z=4.72)$, and 3FGL J1656.2-3303 $(z=2.40)$ using quasi-simultaneous data from the Swift, Nuclear Spectroscopic Telescope Array (NuSTAR) and the Fermi-Large Area Telescope (LAT) and also archival XMM-Newton observations. Other than 3FGL J1656.2-3303, none of the sources were known as $\gamma$-ray emitters, and our analysis of $\sim 7.5 \mathrm{yr}$ of LAT data reveals the first time detection of statistically significant $\gamma$-ray emission from CGRaBS J0225+1846. We generate the broadband spectral energy distributions (SED) of all the objects, centering at the epoch of NuSTAR observations and reproduce them using a one-zone leptonic emission model. The optical-UV emission in all the objects can be explained by radiation from the accretion disk, whereas the X-ray to $\gamma$-ray windows of the SEDs are found to be dominated by inverse Compton scattering off the broad line region photons. All of them host black holes that are billions of solar masses. Comparing the accretion disk luminosity and the jet power of these sources with a large sample of blazars, we find them to occupy a high disk luminosity-jet power regime. We also investigate the X-ray spectral properties of the sources in detail with a major focus on studying the causes of soft X-ray deficit, a feature generally seen in high redshift radio-loud quasars. We summarize that this feature could be explained based on the intrinsic curvature in the jet emission rather than being due to the external effects predicted in earlier studies, such as host galaxy and/or warm absorption.
\end{abstract}

Key words: galaxies: active - galaxies: jets - gamma rays: galaxies

\section{INTRODUCTION}

Blazars constitutes a special class of radio-loud active galactic nuclei (AGNs) in which the relativistic plasma shoots out from the central core of the galaxy, in the form of jets, toward the line of sight to the observer. The flux enhancement due to special relativity and their peculiar orientation (or socalled relativistic beaming) makes blazars visible even at high redshifts $(z>2)$ and thus they can be used to explore the young universe. Blazars are classified as flat spectrum radio quasars (FSRQs) and BL Lac objects based on the rest-frame equivalent width $(\mathrm{EW})$ of their broad optical emission lines, with FSRQs having EW > $>$ A. Among blazars, FSRQs are known up to $z>5$ (e.g., Romani et al. 2004).

The broadband spectral energy distribution (SED) of blazars exhibits a typical double hump structure that is generally explained in terms of synchrotron (from radio to UV-X-rays) and inverse Compton (IC, from X-rays to $\gamma$-rays) emission from a single population of energetic electrons present in the jet (e.g., Ghisellini et al. 1998). It has been found that with increasing luminosity the SED peaks shift to lower frequencies (Fossati et al. 1998). The shift of the synchrotron peak to lower energies leaves the accretion disk radiation (the big blue bump) naked and is observed in the optical-UV SEDs of many FSRQs (see, e.g., Sbarrato et al. 2013a). On the other hand, the shift of the high energy peak from the $\sim \mathrm{GeV}$ to $\sim \mathrm{MeV}$ band makes the high redshift blazars brighter at hard X-rays than in the $\gamma$-ray band and this suggests that hard X-ray telescopes (XRT) are better instruments to constrain the non-thermal jet properties of high redshift blazars (Ghisellini et al. 2010). Thanks to the excellent sensitivity of the recently launched hard X-ray focusing telescope the Nuclear Spectroscopic Telescope Array (NUSTAR; Harrison et al. 2013), it is now possible to explore the rising part of the Compton component of high redshift
FSRQs in detail. Furthermore, the recently released Pass 8 data from the Fermi-Large Area Telescope (Fermi-LAT) have an increased sensitivity at low energies (Atwood et al. 2013) which is important for high redshift objects. Although weak in the $\gamma$-ray window of the SED, a significant $\gamma$-ray detection from high redshift FSRQs will further constrain the location of the IC peak and thus provide a more accurate measurement of their physical properties.

In many high redshift quasars (and also in blazars) a spectral flattening at soft X-ray energies have been noticed $(E \lesssim$ 2-4 keV, see, e.g., Eitan \& Behar 2013 and references therein). Earlier, it was attributed to the presence of absorbing material in the source environment, however, the low level of opticalUV extinction observed in a number of sources is inconsistent with this interpretation. A hypothesis of the presence of a "warm absorber" was also proposed to explain the observed soft X-ray dip (e.g., Worsley et al. 2004a, 2004b). Based on Swift/GRB data, Behar et al. (2011) proposed a diffuse intergalactic medium dominated absorption scenario. However, this feature is more frequently observed in radio-loud quasars than radio-quiet objects (e.g., Eitan \& Behar 2013) and thus requires a connection between $\mathrm{X}$-ray absorption and jet linked activities. More recently, it has been argued that the observed X-ray flattening could be due to low-energy roll-off of the external Compton (EC) process (e.g., Ghisellini et al. 2007; Tavecchio et al. 2007; Foschini 2009).

Here we present the results of a multi-wavelength study of four high redshift FSRQs, namely, S5 0014+81 (hereafter J0014+81; $z=3.37$ ), CGRaBS J0225+1846 (hereafter J0225 $+1846 ; z=2.69$ ), BZQ J1430+4205 (hereafter J1430+4205; $z=4.72$ ), and 3FGL J1656.2-3303 (hereafter J1656-3303; $z=2.40$ ). Our primary goal is to study the broadband physical properties of these high redshift blazars with a major focus on 
their X-ray characteristics. All of them have been observed with NUSTAR along with simultaneous monitoring by Swift (Gehrels et al. 2004), thus providing a unique opportunity to study their $0.3-79 \mathrm{keV} \mathrm{X}$-ray properties. It should be noted that objects $\mathrm{J} 0014+81$ and $\mathrm{J} 0225+1846$ have recently been studied by Sbarrato et al. (2015) using the same data set. Our findings are similar to theirs but we also include the recent XMM-Newton and Fermi-LAT observations and place more emphasis on the X-ray spectral properties. In Section 2, we briefly report the basic information for the four blazars. Section 3 is devoted to the data reduction procedures adopted and the obtained results are presented in Section 4. We discuss our findings in Section 5 and conclude in Section 6. We use a $\Lambda \mathrm{CDM}$ cosmology with the Hubble constant $H_{0}=71$ $\mathrm{km} \mathrm{s}^{-1} \mathrm{Mpc}^{-1}, \Omega_{m}=0.27$, and $\Omega_{\Lambda}=0.73$.

\section{GENERAL PHYSICAL PROPERTIES}

Kuehr et al. (1981) first reported the discovery of J0014+81 and subsequently noted it as an optically luminous quasar (Kuhr et al. 1983). Very long baseline interferometry observations reveal the marginal detection of superluminal patterns (Piner et al. 2012) and also that its optical radiation is not polarized (Wills et al. 2011). Ghisellini et al. (2009) derived its central black hole mass to be as high as $\sim 4 \times 10^{10} M_{\odot}$, however, later it was found to be significantly overestimated (Sbarrato et al. 2015). J0014+81 is included in the 70 months Swift-Burst Alert Telescope (BAT) hard X-ray catalog (Baumgartner et al. 2013) and was observed by NuSTAR on 2014 December 18 and 2015 January 23.

$\mathrm{J} 0225+1846$ was discovered as a flat spectrum radio bright object by Lawrence et al $\left(F_{5 \mathrm{GHz}}=595 \mathrm{mJy} ; 1983\right)$. Subsequently, it was identified as a high redshift blazar in the ROSAT Bright Survey (Schwope et al. 2000). Its is a hard X-ray bright source and is included in the 70 months Swift-BAT catalog. It was observed by NuSTAR on 2014 December 24 and 2015 January 18 along with the simultaneous monitoring from Swift. Moreover, two $\sim 100 \mathrm{ks}$ observations were performed using XMM-Newton (Jansen et al. 2001) in 2013 January. This source is not present in any $\gamma$-ray catalogs but was postulated as a candidate $\gamma$-ray emitting blazar by Healey et al. (2008).

At a redshift of $4.72, \mathrm{~J} 1430+4205$ is a radio-loud $\left(F_{5 \mathrm{GHz}}=230 \mathrm{mJy}\right.$; Helmboldt et al. 2007) and an extremely X-ray luminous blazar (e.g., Fabian et al. 1997, 1998; Hook \& McMahon 1998). Fabian et al. (1999) reported the detection of a significant radio and X-ray flux variability from this source and postulated the presence of an $\sim$ billion solar mass black hole at the center. Later, XMM-Newton observations confirmed the presence of soft X-ray flattening in the X-ray spectrum of this object (Worsley et al. 2004a), a feature typically observed in many high redshift radio-loud quasars (e.g., Page et al. 2005). J1430+4205 was monitored by NuSTAR on 2014 July 14, thereby making it the second most distant source observed by NUSTAR after B2 $1023+25 \quad(z=5.28$; Sbarrato et al. 2013b).

The source J1656-3303 was discovered with the Swift BAT during its first year of survey operation (Okajima et al. 2006). This object lies close to the galactic plane (galactic latitude 6.3). Using the Swift XRT (Burrows et al. 2005) observations, Tueller et al. (2006) suggested it to be an extragalactic source. Later, a detailed multi-wavelength study of this source was performed by Masetti et al. (2008) with a major focus on its optical spectroscopic properties. They confirmed the extragalactic nature of J1656-3303 and concluded it to be a high redshift blazar at $z=2.40$. It is included in the recently released third catalog of Fermi-LAT detected sources (3FGL; Acero et al. 2015). It did not appear in the clean sample of the third catalog of Fermi-LAT detected AGNs (3LAC; Ackermann et al. 2015) because of its close proximity to the galactic plane. This source was simultaneously observed by NuSTAR and Swift on 2015 September 27.

\section{MULTI-WAVELENGTH OBSERVATIONS AND DATA REDUCTION}

\subsection{Fermi-LAT Observations}

Three out of the four sources studied here, J0014+81, J0225 +1846 , and $\mathrm{J} 1430+4205$, are not present in any $\gamma$-ray catalogs. Therefore, by utilizing the availability of recently released Pass 8 data from Fermi-LAT, we not only study the $\gamma$-ray properties of the known $\gamma$-ray emitter J1656-3303 but also search for the possible detection of the remaining three sources in the LAT energy range. We consider the first 89 months of Fermi-LAT data (MJD 54683-57391 or 2008 August 4 to 2016 January 4) and follow the standard procedure as described in the online documentation. ${ }^{4}$ In the energy range of 0.1 $-300 \mathrm{GeV}$, we select only events belonging to the SOURCE class (corresponding to evclass $=128$ ) and use a relational filter "DATA_QUAL > 0," \& "LAT_CONFIG==1" to select good time intervals. To avoid the contamination from Earth limb $\gamma$-rays, we reject all the events with a zenith angle larger than $90^{\circ}$. We define the region of interest (ROI) as a circle of $15^{\circ}$ radius centered at the target source to perform the likelihood fitting. All the objects lying within the ROI and present in the 3FGL catalog are considered, and the spectral parameters of those lying within $10^{\circ}$ are left free to vary during the fitting. The parameters of the remaining sources are fixed to the 3FGL catalog values. We use a binned likelihood method and compute the significance of the $\gamma$-ray signal by means of the maximum likelihood (ML) test statistic TS $=2 \Delta \log (\mathcal{L})$ where $\mathcal{L}$ represents the likelihood function, between models with and without a point source at the position of the source (Mattox et al. 1996). To search for the presence of additional faint objects that could be present in the data but not in the 3FGL catalog, we generate the residual TS maps, after performing the first round of likelihood fitting. If present, these newly detected objects are then modeled with a powerlaw model and another round of fitting is performed. We generate another residual TS map to ascertain that no sources are left to model. We then removed all the sources having TS $<25$ to perform further temporal and spectral studies. To generate both a light curve and a spectrum, the source is considered to be detected if TS $>9$. For bins with $\mathrm{TS}<9$ and or $\Delta F_{\gamma} / F_{\gamma}>0.5$, where $\Delta F_{\gamma}$ is the error estimate in the flux $F_{\gamma}$, we calculate a $2 \sigma$ upper limit. In this work, all the errors associated with the LAT data analysis are $1 \sigma$ statistical uncertainties, unless specified.

\subsection{NUSTAR Observations}

The blazar J0014+81 was monitored two times by NuSTAR, on 2014 December 21 (obs ID 60001098002) and 2015 January 23 (obs ID 60001098004) for a net exposure of $36.4 \mathrm{ks}$ and $31 \mathrm{ks}$, respectively. J0225+1846 was also observed

\footnotetext{
4 http://fermi.gsfc.nasa.gov/ssc/data/analysis/documentation/
} 
twice, on 2014 December 24 (obs ID 60001101002) and 2015 January 18 (obs ID 60001101004) for net exposure times of 32.0 and $37.5 \mathrm{ks}$, respectively. $\mathrm{J} 1430+4205$ was monitored on 2014 July 14 (obs ID 60001103002) for $49.2 \mathrm{ks}$ and J1656-3303 was observed by NuSTAR on 2015 September 27 (obs ID 60160657002) for a net exposure time of $21.1 \mathrm{ks}$.

The downloaded data sets for Focal Plane Module A and Focal Plane Module B were analyzed using the NuSTAR Data Analysis Software (NUSTARDAS) version 1.5.1. The task nupipeline was used to clean and calibrate the events' data files using standard filtering criteria and NuSTARCALDB, updated on 2015 October 8. Source and background spectra, along with the ancillary and response matrix files, were generated using the task nuproducts. To generate the source spectrum, we selected the source region as a circle of $30^{\prime \prime}$ radius centered at the target source. The background spectrum was extracted from a circular region of $70^{\prime \prime}$ on the same chip, free from source contamination. We binned the source spectrum to have a signal-to-noise ratio of 6 , and oversampled the spectra by a factor of 3 .

\subsection{XMM-Newton Observations}

J0014+81 was observed with XMM-Newton on 2001 August 23, for a net exposure of $42.9 \mathrm{ks}$ (obs ID 0112620201). J0225+1846 was observed three times, once in 2003 (July 25, obs ID 0150180101) and twice in 2013 (January 13 and 15, obs IDs 0690900101 and 0690900201), for exposures of 22.2, 108.0, and $96.7 \mathrm{ks}$, respectively. J1430+4205 was also observed three times, on 2002 December 09 (obs ID 0111260101), 2003 January 17 (obs ID 0111260701), and 2005 June 05 (obs ID 0212480701) for exposures of 18.9, 14.6 and $19.7 \mathrm{ks}$. Finally, J1656-3303 was observed on 2009 September 11 (obs ID 0601741401) for net exposure of $22.6 \mathrm{ks}$.

We use the XMM-Newton Science Analysis Software version 15.0.0 to reduce the data. We use the rgsproc task to reduce the RGS spectra and the epproc task to produce the EPIC-pn event files, which we then filter for high background using evselect. We extract source spectra from $30^{\prime \prime}$ circular regions, and background spectra from $60^{\prime \prime}$ circular regions on the same chip, avoiding contaminating sources. The spectra are binned using specgroup to a signal-to-noise ratio of 6 , after background subtraction, and to oversample the data by a factor of 3 .

\subsection{Swift Observations}

Swift observations were carried out in conjunction with NUSTAR monitoring for all four blazars. Swift observed J0014 +81 on 2014 December 21 (obs ID 00080003001, net exposure $6.5 \mathrm{ks}$ ) and 2015 January 23 (obs ID 00080003002 , net exposure $6.6 \mathrm{ks}$ ). The source $\mathrm{J} 0225+1846$ was monitored on 2014 December 24 (obs ID 00080243001, net exposure $4.9 \mathrm{ks}$ ) and 2015 January 18 (obs ID 00080243002, net exposure $5.1 \mathrm{ks}$ ). Swift observed J1430+4205 on 2014 July 13 (obs ID 00080752002, net exposure $7.5 \mathrm{ks}$ ) and J1656 -3303 on 2015 September 27 (obs ID 00081202001, net exposure $6.8 \mathrm{ks}$ ).

Swift-XRT observations of all the sources were carried out using the most sensitive photon counting mode (standard grade selection 0-12). The event files were calibrated and cleaned with the task xrtpipeline and using the latest calibration files. The calibrated and cleaned event files were summed using xselect and the resultant summed event files were used to extract the energy spectrum. We selected the source region as a circle of 55" radius, and events for the background spectra were extracted from an annular ring with inner and outer radii of $110^{\prime \prime}$ and $210^{\prime \prime}$, respectively, both centered at the position of the source of interest. We combined the exposure maps using the tool ximage, which takes into account CCD defects and point-spread function losses, and generated the ancillary response files using xrtmkarf. The source spectra were binned to have a signal-to-noise ratio of 6 .

The Swift UltraViolet Optical Telescope (UVOT; Roming et al. 2005) has observed the sources of interest in all the six filters ( $V, B, U, W 1, M 2$, and $W 2$ ). To improve the signal-tonoise ratio, we add UVOT snapshots using the tool uvotimsum. The source counts are extracted from a circular region of $5^{\prime \prime}$ centered at the source of interest, while the background is selected as a circle of $30^{\prime \prime}$ radius from a nearby source-free region. The task uvotsource is used to extract the magnitudes which are then corrected for Galactic reddening (Schlafly \& Finkbeiner 2011). The de-absorbed magnitudes are converted to flux units using the zero point and calibrations of Breeveld et al. (2011).

\section{RESULTS}

\subsection{Gamma-ray Properties}

None of the blazars under consideration, except J1656-3303, are present in any $\gamma$-ray catalogs. Therefore, using the good quality Pass 8 LAT data, which are more sensitive at low energies, we search for a possible $\gamma$-ray signal from all the four sources. This is done by performing an average analysis of $\sim 7.5 \mathrm{yr}$ of LAT data. Other than J1656 -3303 , we find a statistically significant $\gamma$-ray emission from $\mathrm{J} 0225+1846$ (TS $\approx 188, \sim 13 \sigma$ detection). To ensure that the observed $\gamma$-ray emission is associated with target blazars and not with any other unmodeled objects lying close to the sources of interest, we generate their residual TS maps and show them in Figure 1. As can be seen, the residual TS map of J0225 +1846 suggests the presence of a few unmodeled sources (with TS $>25$ ) and we properly consider them in the analysis. The residual TS map of the same field, after taking unmodeled objects into account, verifies that no other sources (with TS $>25$ ) are left to model. The $\gamma$-ray spatial location of J0225 +1846 is optimized using the tool gtfinds $r \mathrm{c}$ and derived as R.A., decl. $=36^{\circ} .177,18^{\circ} .859(\mathrm{~J} 2000)$ with a $95 \%$ error circle radius of $0^{\circ} .21$ degrees. We cross-check in the NRAO VLA Sky Survey (Condon et al. 1998) for the presence of additional radio sources within the $95 \%$ contour and find a total of ten sources with J0225+1846 being the brightest $\left(F_{1.4 \mathrm{GHz}}=460.8 \mathrm{mJy}\right)$. Then, we search in the NASA/IPAC Extragalactic Database ${ }^{5}$ for multi-wavelength counterparts of these radio sources and find no information for any objects other than $\mathrm{J} 0225+1846$. This indicates the close association of J0225+1846 with the $\gamma$-ray emitter. Furthermore, the TS map of J1656-3303 does not reveal any unmodeled sources and therefore the model used during the analysis is an accurate representation of the observed $\gamma$-rays, with no new $\gamma$-ray point source being present. Apart from these, LAT data analysis of $\mathrm{J} 0014+81$ and $\mathrm{J} 1430+4205$ resulted in $\mathrm{TS}=1.47$ and 5.62,

5 http://ned.ipac.caltech.edu/ 

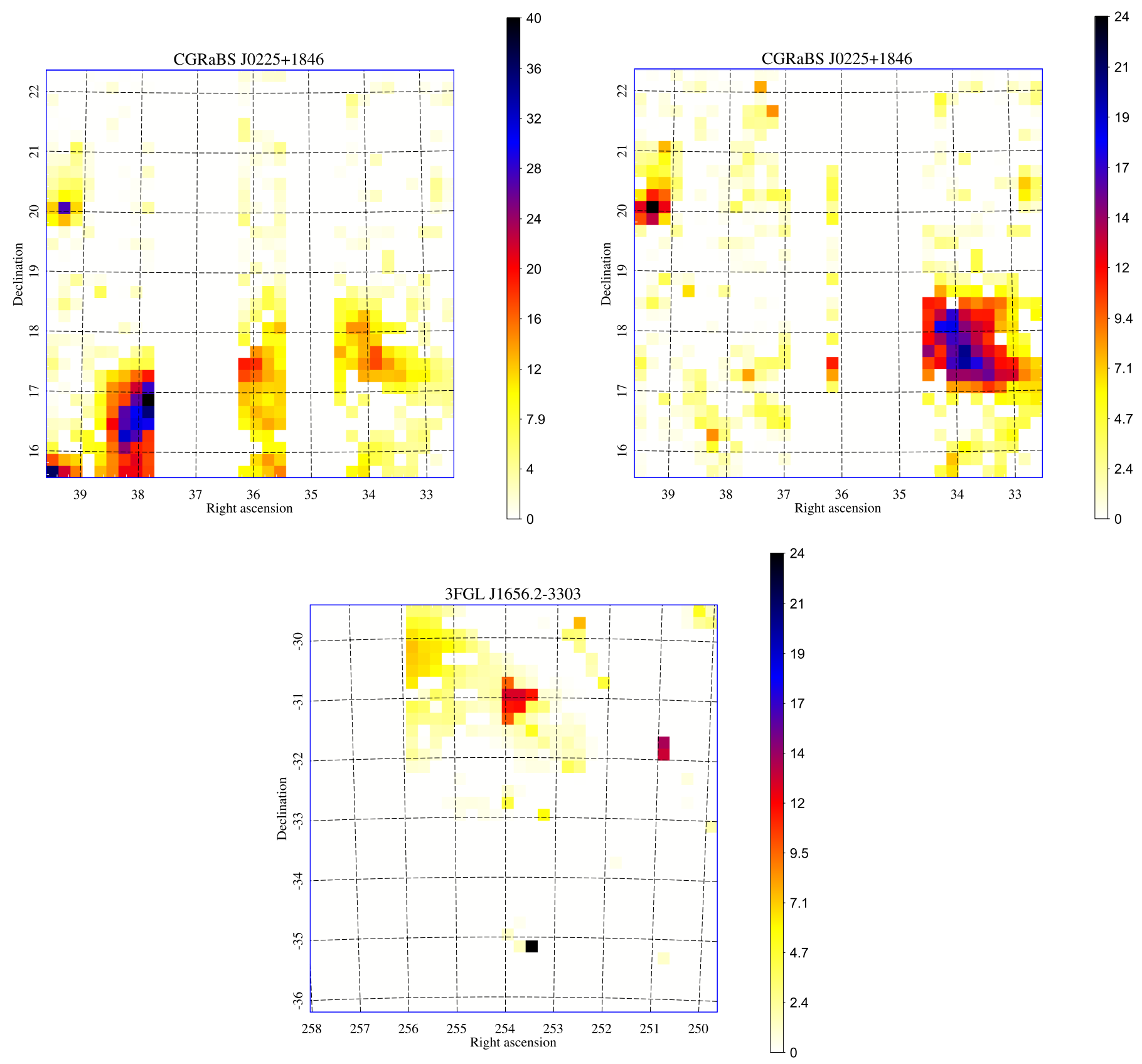

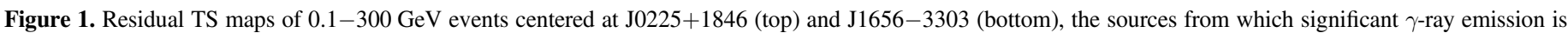

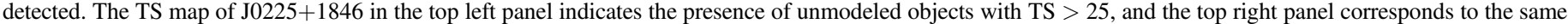

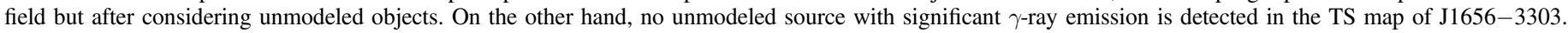

respectively. This suggests the non-detection of J0014+81 and only a marginal $\sim 2 \sigma$ detection of J1430 4205 in the $\gamma$-ray band. Two models, a power law $\left(N(E)=N_{0}\left(E / E_{0}\right)^{-\Gamma_{\gamma}}\right.$, where $\Gamma_{\gamma}$ is the photon index) and a logParabola $\left(N(E)=N_{0}\left(E / E_{\text {pivot }}\right)^{-\alpha-\beta \log \left(E / E_{\text {pivot }}\right)}\right.$, where $\alpha$ is the photon index at $E_{\text {pivot }}, \beta$ is the curvature index, and $E_{\text {pivot }}$ is pivot energy fixed at $300 \mathrm{MeV}$ ), are applied to the $\gamma$-ray spectra of $\mathrm{J} 0225+1846$ and J1656-3303. The results of the average LAT data analysis are presented in Table 1.

We also study the $\gamma$-ray temporal behavior of J0225+1846 and $\mathrm{J} 1656-3303$. This is achieved by generating monthly binned $\gamma$-ray light curves which we show in Figure 2. Both of them are faint in $\gamma$-rays and only a moderate brightening of
$\mathrm{J} 0225+1846$ is seen around the beginning of 2013. On the other hand, J1656-3303 is occasionally detected by LAT without any flaring activity.

\subsection{X-Ray Properties}

We fit the NuSTAR $(3-79 \mathrm{keV})$, Swift-XRT $(0.3-10 \mathrm{keV})$, and XMM-Newton $(0.3-10 \mathrm{keV}$ for EPIC-pn and $0.5-2 \mathrm{keV}$ for RGS) spectra using XSPEC (Arnaud 1996) version 12.9.0j. In all cases we include Galactic absorption, taken from the online tool of Willingale et al. (2013), of $2.19 \times 10^{21} \mathrm{~cm}^{-2}$ for J0014 $+81,1.68 \times 10^{21} \mathrm{~cm}^{-2}$ for J0225+1486, $1.20 \times 10^{20} \mathrm{~cm}^{-2}$ for $\mathrm{J} 1430+4205$, and $3.35 \times 10^{21} \mathrm{~cm}^{-2}$ for $\mathrm{J} 1656-3303$. We use 
Table 1

Results of the LAT Data Analysis of High Redshift Blazars Studied in this Work, Covering the First $~ 7.5$ yr of Fermi Operation

\begin{tabular}{|c|c|c|c|c|c|c|}
\hline $\begin{array}{l}\text { Name } \\
{[1]}\end{array}$ & $\begin{array}{c}\text { Model } \\
{[2]}\end{array}$ & $\begin{array}{c}F_{0.1-300 \mathrm{GeV}} \\
\text { [3] }\end{array}$ & $\begin{array}{c}\Gamma_{0.1-300 \mathrm{GeV}} / \alpha \\
{[4]}\end{array}$ & $\begin{array}{c}\beta \\
{[5]}\end{array}$ & $\begin{array}{l}\text { TS } \\
{[6]}\end{array}$ & $\begin{array}{c}\mathrm{TS}_{\text {curve }} \\
{[7]}\end{array}$ \\
\hline \multirow[t]{2}{*}{ J0014+81 } & PL & 0.15 (UL) & $\cdots$ & $\cdots$ & 1.47 & $\cdots$ \\
\hline & LP & $\ldots$ & $\ldots$ & $\cdots$ & $\ldots$ & $\cdots$ \\
\hline $\mathrm{J} 0225+1846$ & LP & $2.49 \pm 0.25$ & $2.86 \pm 0.14$ & $0.45 \pm 0.21$ & 195.51 & 9.82 \\
\hline \multirow[t]{2}{*}{$\mathrm{J} 1430+4205$} & PL & $0.42(\mathrm{UL})$ & $\ldots$ & $\ldots$ & 5.62 & $\cdots$ \\
\hline & LP & $\ldots$ & $\ldots$ & $\cdots$ & $\cdots$ & $\cdots$ \\
\hline
\end{tabular}

Note. Column [1]: object name; Column [2]: model used (PL: power law, LP: logParabola); Column [3]: integrated $\gamma$-ray flux $(0.1-300 \mathrm{GeV})$, in units of 10 $\mathrm{ph} \mathrm{cm}^{-2} \mathrm{~s}^{-1}$, UL corresponds to the $2 \sigma$ upper limit; Columns [4] and [5]: spectral parameters (see definitions in the text); Column [6]: test statistic; and Column [7]: curvature of test statistic.

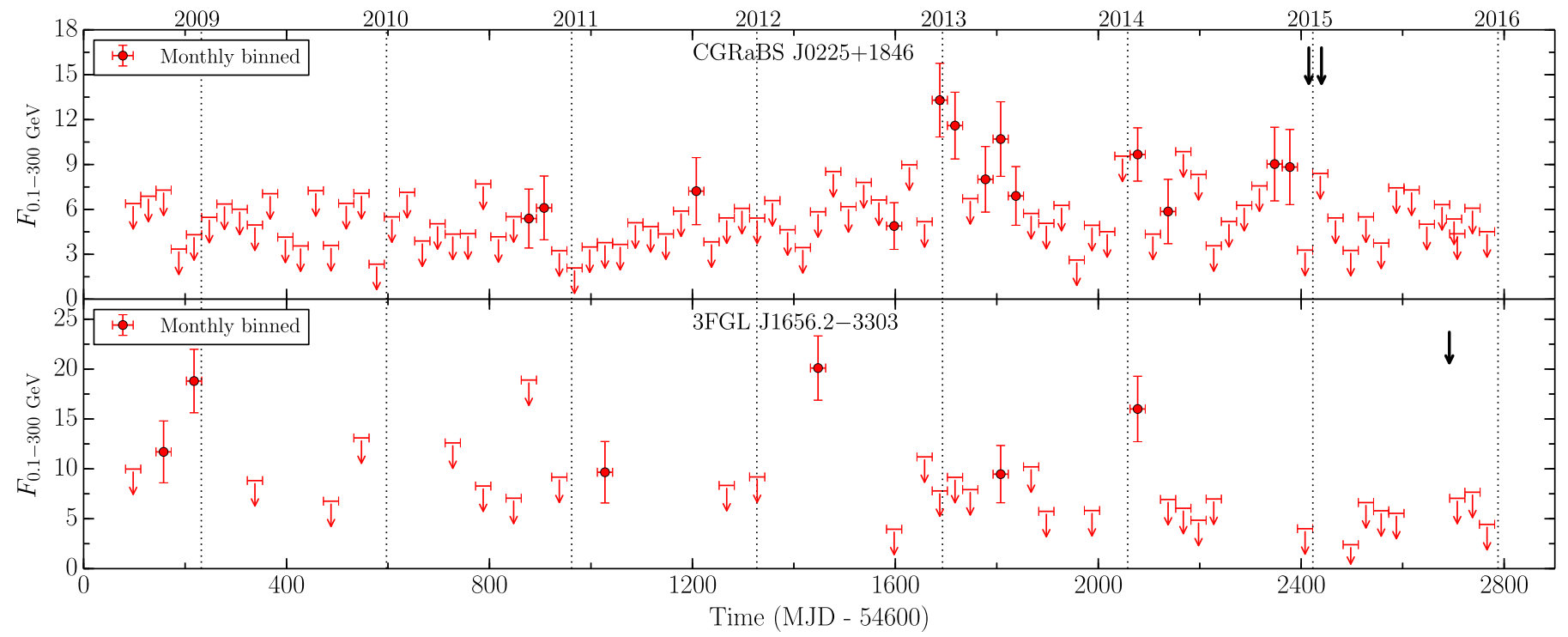

Figure 2. Temporal behavior of J0225+1846 (top) and J1656-3303 (bottom) in the $0.1-300 \mathrm{GeV}$ energy range, as observed by Fermi-LAT. The fluxes are in units of $10^{-8} \mathrm{ph} \mathrm{cm}^{-2} \mathrm{~s}^{-1}$. Red downward arrows represent $2 \sigma$ flux upper limits, and black downward arrows denote the time of NuSTAR monitoring. Note that time bins with TS $<1$ are not shown. Vertical dotted lines correspond to the beginning of the calendar years.

wilm abundances (Wilms et al. 2000) and vern cross sections (Verner et al. 1996).

\subsubsection{Broadband $0.3-79 \mathrm{keV}$ Spectra}

The most useful spectra for determining the nature of the change in slope in the X-ray band are the simultaneous Swift-XRT and NuSTAR spectra, which cover the energy range from $\sim 0.3-79 \mathrm{keV}$. We fit the same three models to each spectrum: a power law absorbed by Galactic absorption only (M1, tbabs * powerlaw in XSPEC); a power law absorbed by Galactic absorption and absorption intrinsic to the source (M2, tbabs * ztbabs * powerlaw); and a broken power law, absorbed by Galactic absorption only (M3, tbabs * bknpower). These correspond to a spectrum with no curvature, a spectrum where the curvature is due to absorption of the source by the host galaxy, and a spectrum where the curvature is intrinsic. Two of the sources $(\mathrm{J} 0014+81$ and $\mathrm{J} 0225+1846)$ were observed twice with NuSTAR and Swift. In the case of J0014+81 the two spectra are indistinguishable, so we fit them simultaneously with the same model. For J0225+1846 the observations are further apart and show significant spectral evolution. We therefore fit them simultaneously but allow the model parameters to vary between the two observations.

The best fit parameters for each model and object are given in Table 2 and the fit residuals are shown in Figure 3. In each case, the quality of the fit improves from M1 to M3, with the absorbed power law preferred over the unabsorbed power law, and the broken power law better still. The improvement in $\chi^{2}$ from switching from M2 to M3 is marginal for $\mathrm{J} 0014+81$, $\mathrm{J} 1430+4205$, and J1656-3303, so we check the significance using an F-test. We find chance probabilities of 0.0003, 0.011, and 0.008 , respectively (in all cases, the improvement obtained by switching from $\mathrm{M} 1$ to $\mathrm{M} 2$ or $\mathrm{M} 3$ is significant at $\gg 3 \sigma$ ).

We also consider the effect of ionized absorption (warm absorber) models. We replace the neutral absorber in M2 with an ionized absorber (modeled using an XSTAR grid), and re-fit the data for each source. This improves the fit significantly relative to the neutral absorber only in $\mathrm{J} 0225+1846$ $\left(\chi_{\nu}^{2}=1.04\right)$, with a column density of $N_{\mathrm{H}}=5.5_{-0.9}^{+1.7} \times 10^{23}$, 
Table 2

Fit Parameters From the Joint NuSTAR-XRT Spectra

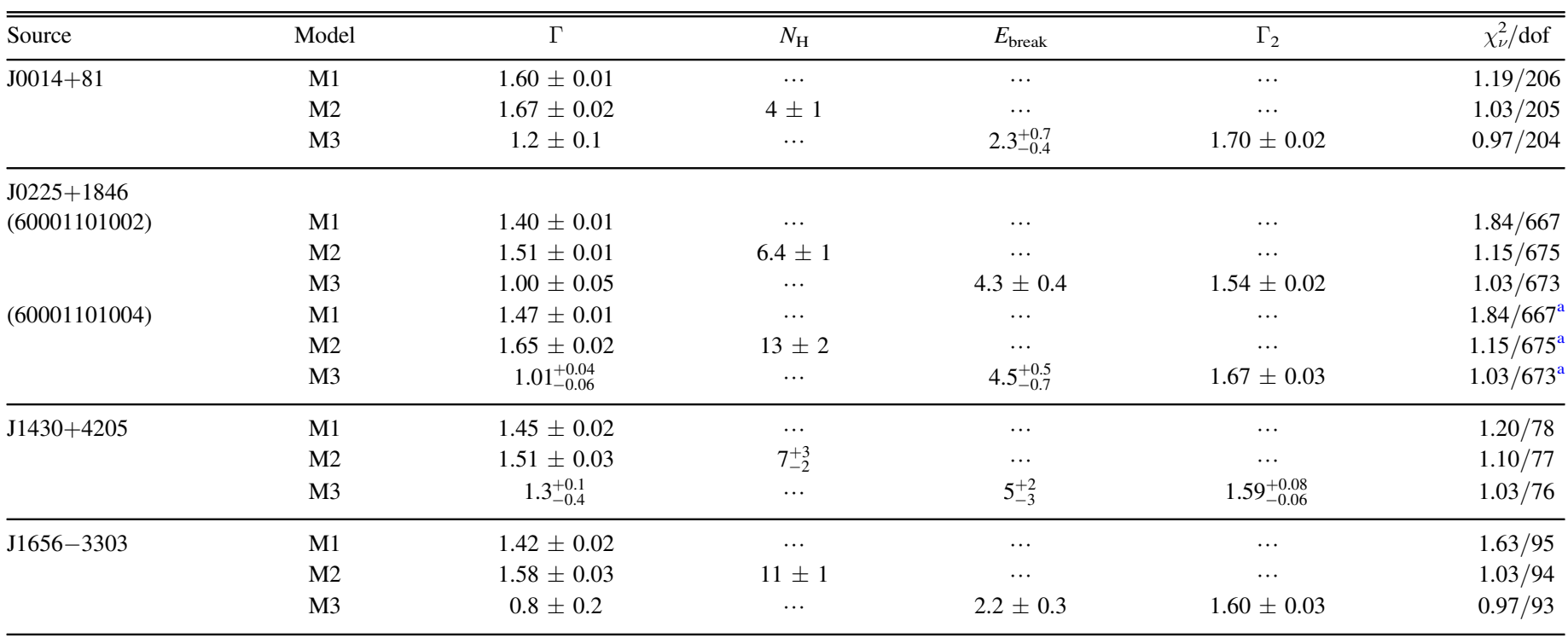

Notes. Column densities are in units of $10^{22} \mathrm{~cm}^{-2}$.

${ }^{\mathrm{a}}$ The $\chi^{2}$ values are identical for the two observations of $\mathrm{J} 0225+1486$ because the spectra are fit simultaneously.

and ionization of $\log (\xi)=2.8 \pm 0.1$ (the spectral variability between the two observations is mostly in the power law).

\subsubsection{XMM-Newton}

We next fit the XMM-Newton data for each object. We fit the EPIC-pn spectrum for each source, fitting simultaneously but allowing parameters to vary independently when there are multiple spectra. For J0225+1846, there are also highresolution RGS spectra, which we include in the fit with model parameters tied to those of the corresponding EPIC-pn spectrum. For this fit we use the higher resolution tbnew (Wilms et al. 2000).

As these observations are not taken simultaneously with a hard X-ray instrument the energy band is significantly narrower, making it more difficult to detect spectral curvature. As such, additional curvature is not significantly detected in one source, J0014+81. This spectrum is well fit with a simple power-law model $\left(\mathrm{M} 1, \chi^{2} / \mathrm{dof}=112 / 116\right)$ and the fit is not significantly improved by either additional absorption or a spectral break.

The other three sources show evidence of spectral curvature, with a significant improvement in the fit from M1 to M2 or M3. There is only a significant improvement from M2 to M3 in the case of $\mathrm{J} 1656-3303(p=0.004)$. For $\mathrm{J} 0225+1486$ the improvement is marginal $(p=0.1)$ and for $\mathrm{J} 1430+4205$ the fit is slightly worse with M3 than M2, although not significantly so. The data and residuals to each model are shown in Figure 4 and the parameters are given in Table 3.

\subsection{Spectral Energy Distributions}

\subsubsection{Model Setup}

We use the simple one-zone synchrotron and IC emission model fully described in Ghisellini \& Tavecchio (2009) and here we discuss it in brief. We assume the emission region is a sphere of radius $R_{\mathrm{blob}}$, located at a distance $R_{\text {diss }}$ from the central black hole, and moving with a bulk Lorentz factor $\Gamma$. The emitting region is filled with relativistic electrons having a smooth broken power-law energy distribution of the following type

$$
Q(\gamma)=Q_{0} \frac{\left(\gamma_{\mathrm{b}}\right)^{-n 1}}{\left(\gamma / \gamma_{\mathrm{b}}\right)^{n 1}+\left(\gamma / \gamma_{\mathrm{b}}\right)^{n 2}} .
$$

The size of the emission region is constrained by assuming it covers the entire jet cross-section with a semi-opening angle of the jet of $0.1 \mathrm{rad}$. The magnetic field is considered as tangled and uniform in the emission region. We consider several sources of thermal radiation external to the jet: (i) direct radiation from the accretion disk; (ii) the X-ray corona sandwiching the accretion disk, reprocessing a fraction $f_{\text {cor }}$ of the accretion disk luminosity $\left(L_{\text {disk }}\right)$, and having a cut-off power-law spectrum $\left(L_{\text {cor }}(\nu) \propto \nu^{-\alpha_{\text {cor }}} \exp \left(-\nu / \nu_{\mathrm{c}}\right)\right)$; (iii) the broad line region (BLR), assumed to reprocess a fraction $f_{\mathrm{BLR}}$ of $L_{\text {disk }}$ from a spherical shell located at a distance $R_{\mathrm{BLR}}=10^{17} L_{\text {disk, } 45}^{1 / 2} \mathrm{~cm}$, where $L_{\mathrm{disk}, 45}$ is the disk luminosity in units of $10^{45} \mathrm{erg} \mathrm{s}^{-1}$; (iv) and the IR emission from a dusty torus located at a distance $R_{\text {torus }}=10^{18} L_{\text {disk, } 45}^{1 / 2} \mathrm{~cm}$ and which re-emits a fraction $f_{\text {torus }}$ of the accretion luminosity. The spectra of both the BLR and the torus are assumed to be a blackbody peaking at the rest-frame frequency of the Ly $\alpha$ line (e.g., Tavecchio \& Ghisellini 2008) and $T_{\text {torus }}$, respectively, where $T_{\text {torus }}$ is the characteristic temperature of the torus. In the comoving frame, we appropriately evaluate the radiative energy densities of these components and used them to derive the EC spectrum, and then transform into observer frame. We estimate the power that the jet carries in the form of the magnetic field $\left(P_{\mathrm{mag}}\right)$, radiation $\left(P_{\mathrm{rad}}\right)$, relativistic electrons $\left(P_{\text {ele }}\right)$, and cold protons $\left(P_{\text {kin }}\right)$. The last quantity, i.e., the kinetic jet power, is derived by assuming an equal number density of protons and relativistic electrons (e.g., Celotti \& Ghisellini 2008).

The flux produced by the accretion disk is estimated by assuming a standard Shakura \& Sunyaev (1973) disk having a 
J0014+81

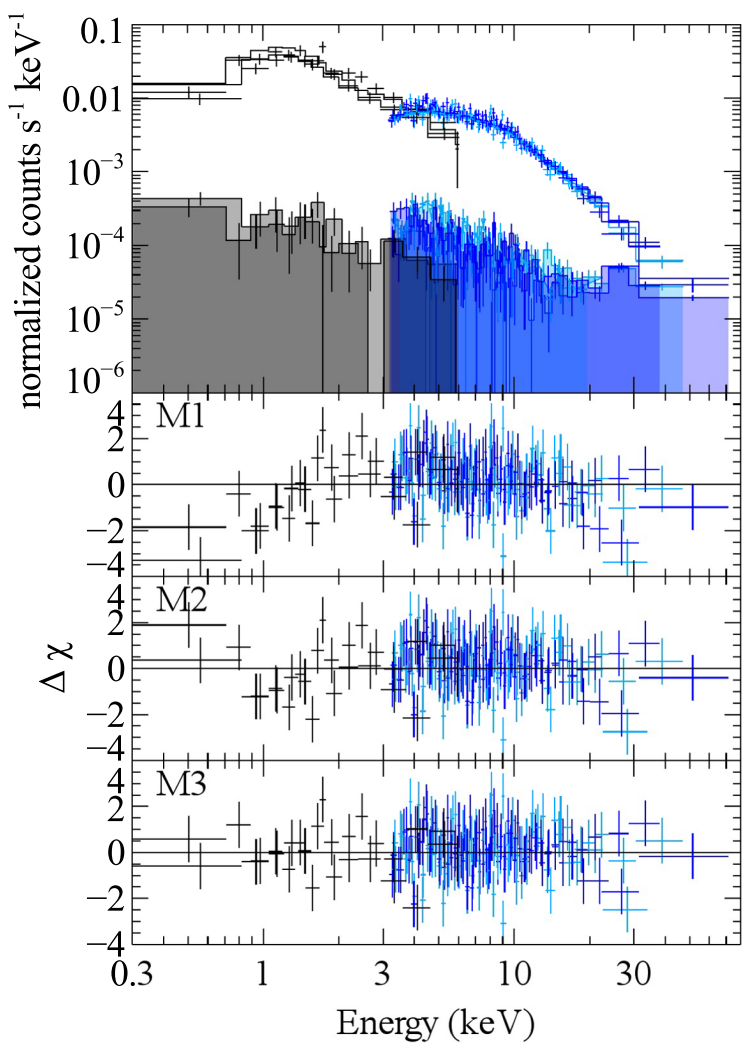

$\mathrm{J} 1430+4205$

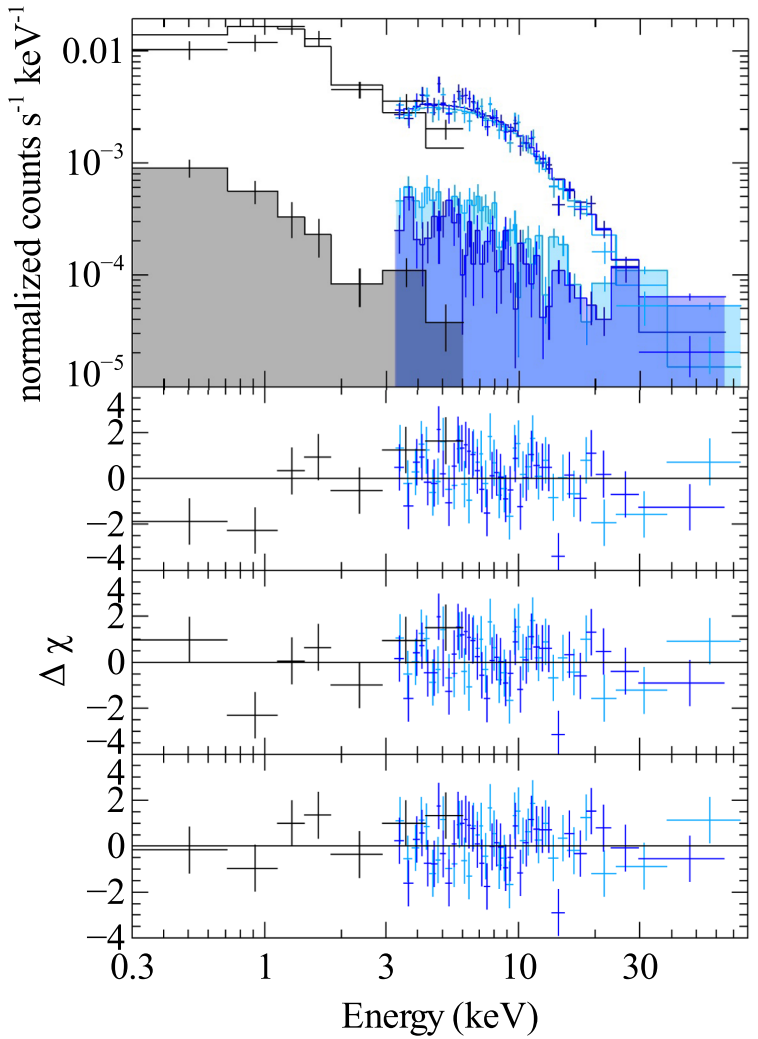

J0225+1846

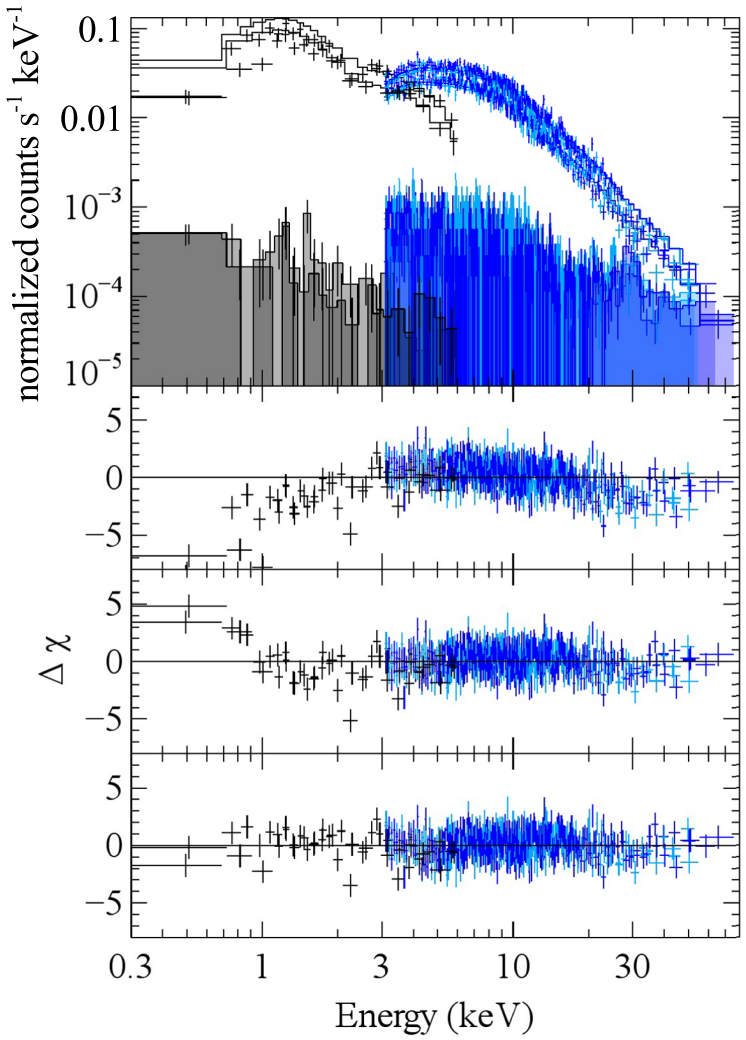

J1656-3303

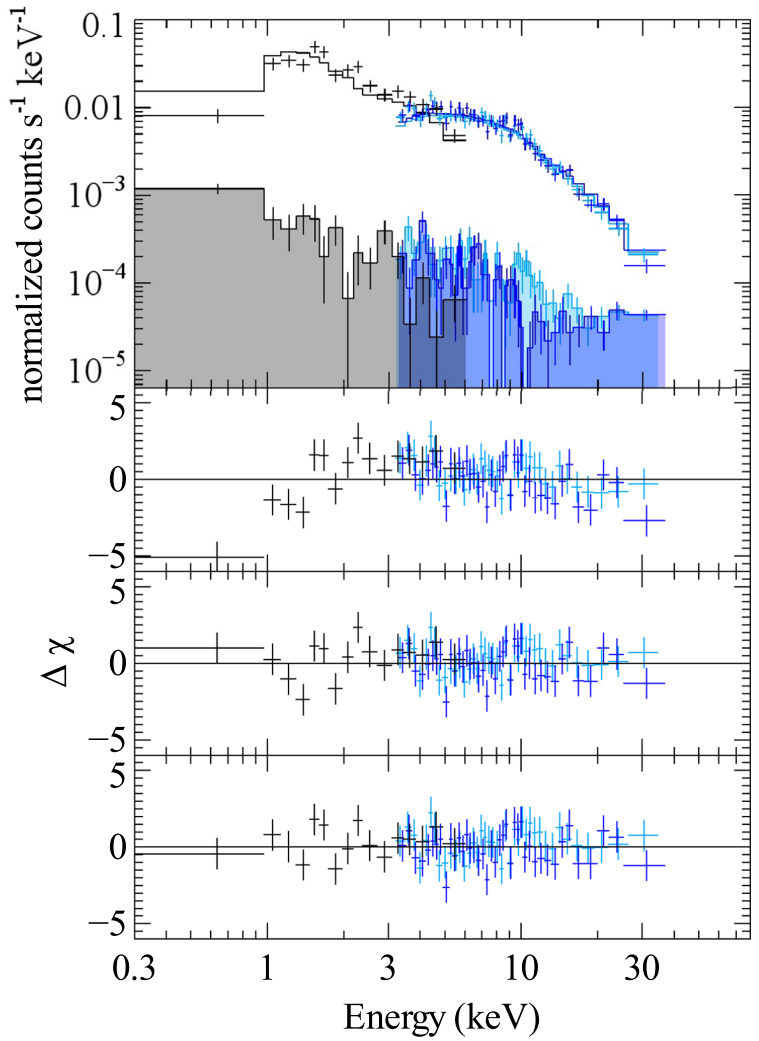

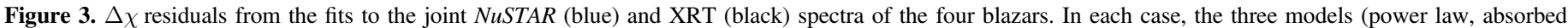

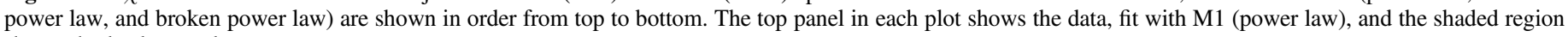
shows the background spectrum. 

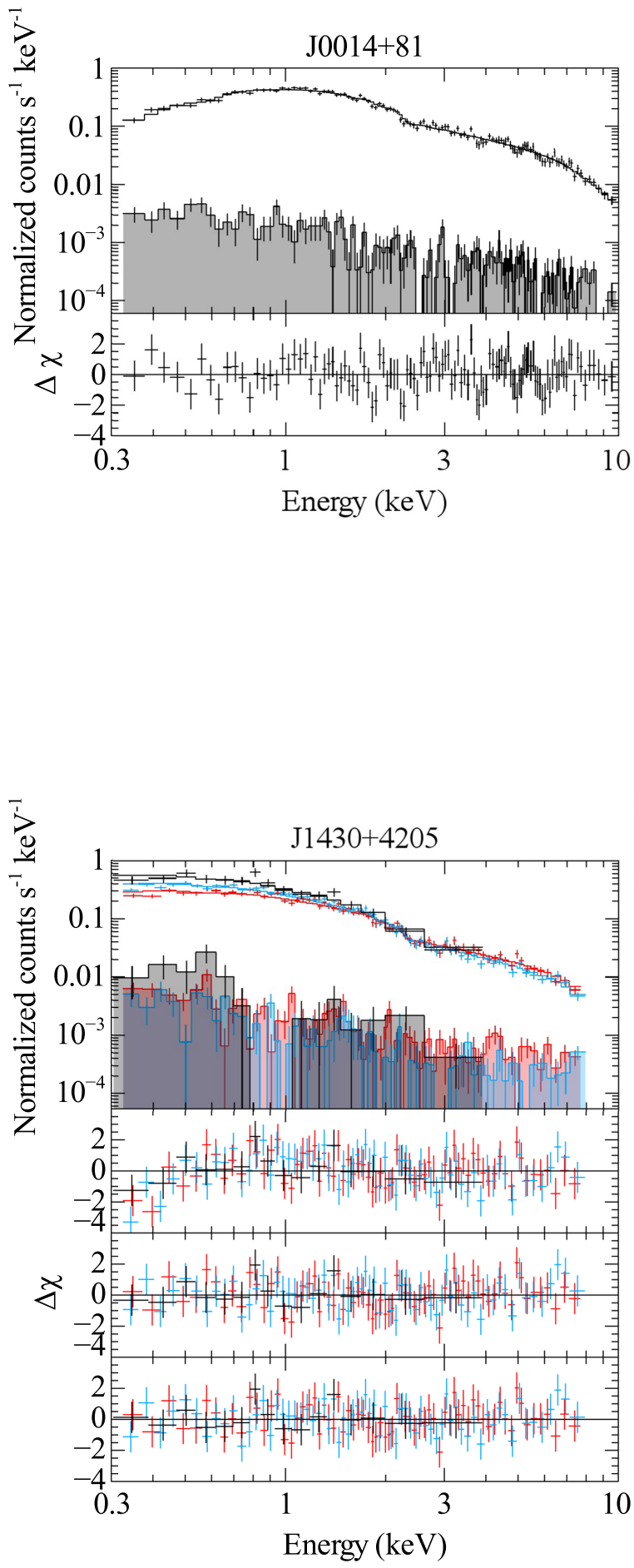
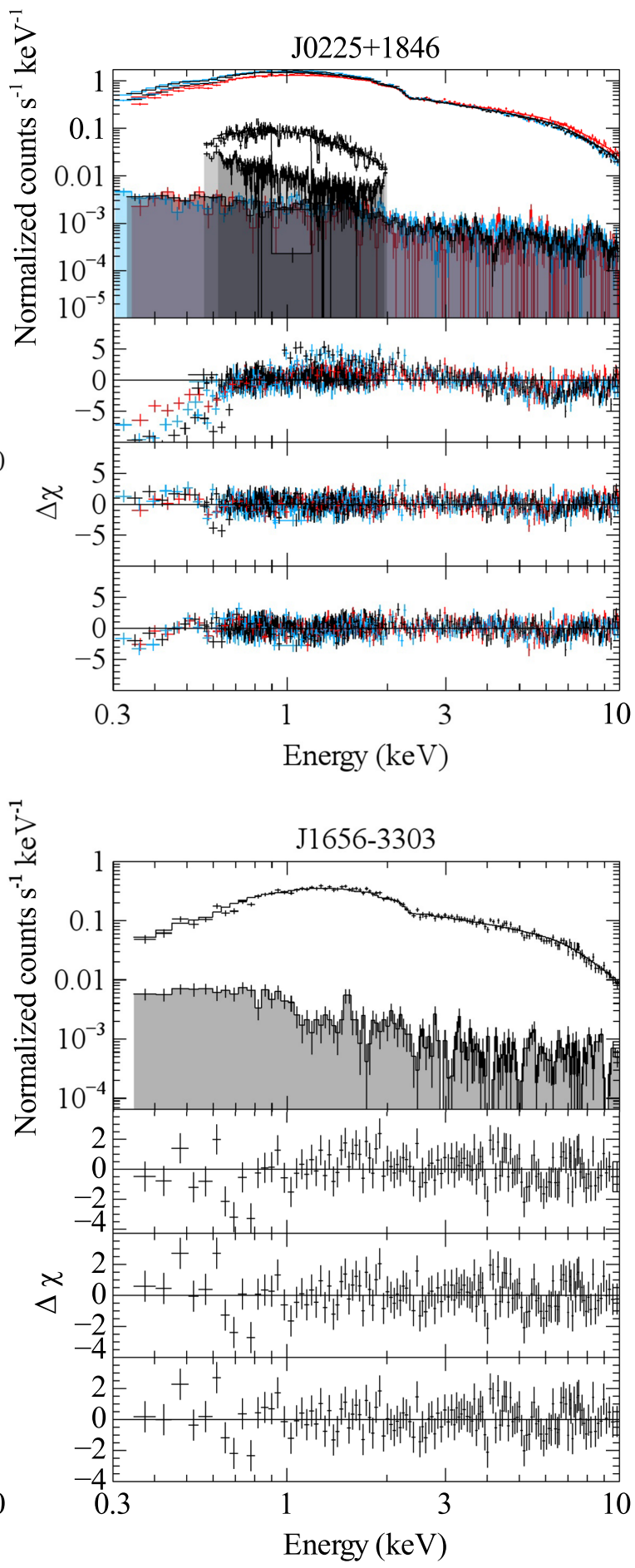

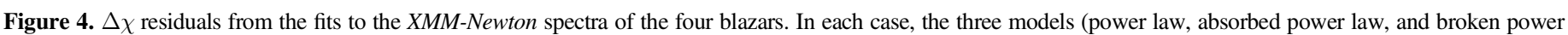

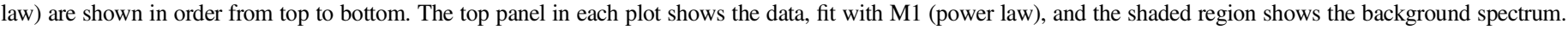

multi-temperature radial profile as follows (Frank et al. 2002)

$$
T=\frac{3 R_{\mathrm{Sch}} L_{\mathrm{disk}}}{16 \pi \eta_{\mathrm{acc}} \sigma_{\mathrm{MB}} R^{3}}\left[1-\left(\frac{3 R_{\mathrm{Sch}}}{R}\right)^{1 / 2}\right]^{1 / 4},
$$

where $R_{\mathrm{Sch}}$ is the Schwarzschild radius and $\eta_{\mathrm{acc}}$ is the accretion efficiency. This is used to reproduce the thermal radiation (the big blue bump) observed at optical-UV energies and it constrains both $L_{\text {disk }}$ and the central black hole mass of the source. There are only two parameters to be fitted, the accretion rate $\dot{M}_{\text {acc }}$ and the black hole mass, once we assume a typical value of the accretion efficiency $\left(\eta_{\text {acc }}=10 \%\right)$. The former can be derived from the intrinsic accretion disk luminosity $L_{\text {disk }}=\eta_{\text {acc }} \dot{M}_{\text {acc }} c^{2}$ with $L_{\text {disk }}$ being an observable parameter (provided the peak of the big blue bump is visible in the SED). This leaves only black hole mass as a free parameter and can be calculated from the optical-UV SED fitting. 
Table 3

Fit Parameters from the XMM-Newton Spectra

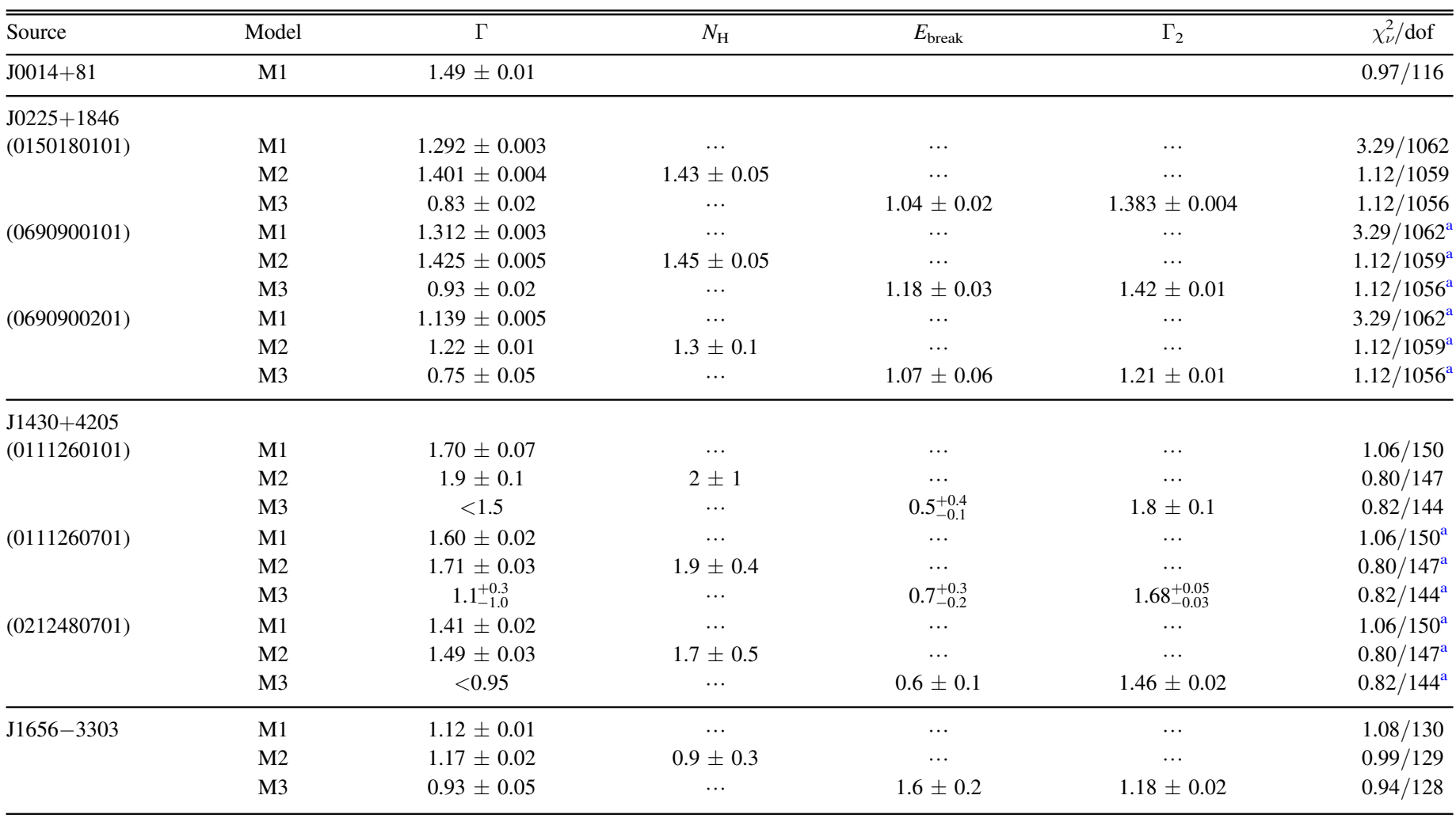

Notes. Column densities are in units of $10^{22} \mathrm{~cm}^{-2}$

${ }^{\mathrm{a}}$ The $\chi^{2}$ values are identical for the three observations of $\mathrm{J} 0225+1486$ and $\mathrm{J} 1430+4205$ because the spectra are fit simultaneously. Additionally, for the J0225+1486 source we include the RGS data and use tbnew instead of tbabs.

\subsubsection{SED Modeling Results}

The broadband SEDs of all the four blazars are generated using quasi-simultaneous data from Swift, NuSTAR, and Fermi-LAT. None of the sources are detected by LAT even at the $3 \sigma$ level in the month of NUSTAR observations and therefore we calculate their respective $2 \sigma$ upper limits. The generated SEDs are reproduced by the model presented in Section 4.3.1. For modeling of the SEDs, we adopt the following assumptions: the spectral shape of the X-ray corona emission is assumed to be flat $\left(\alpha_{\text {cor }}=1\right)$ and the cut-off energy is fixed at $150 \mathrm{keV}$ (Ghisellini \& Tavecchio 2009). Fractions of the accretion disk luminosity re-emitted by the X-ray corona, the BLR, and the dusty torus are considered as $f_{\text {cor }}=0.3$, $f_{\mathrm{BLR}}=0.1$, and $f_{\text {torus }}=0.5$, respectively. The results of the SED generation and modeling are presented in Figure 5 and the associated modeling parameters are given in Tables 4 and 5 .

\section{DISCUSSION}

A study of the high redshift blazars offers a unique opportunity to understand the physical properties of jetted sources at the extreme end of the accretion disk luminosity and the jet power. Since blazars emit a major fraction of their bolometric luminosity in the form of high energy X-ray and $\gamma$-ray radiation, it is important to have good quality sensitive monitoring at these energies. In this regard, the observations from facilities like Fermi-LAT, NuSTAR, and
XMM-Newton are crucial for learning about the radiative processes powering the jets of these distant sources.

\subsection{Gamma-ray Emission}

The high redshift blazars are, in general, weak in the $\gamma$-ray band because of the $k$-correction effect (for increasing redshifts) and also due to intrinsic shift of the IC peak at lower frequencies as their bolometric luminosity increases (e.g., Ghisellini et al. 1998). Three out of the four sources studied here were never detected in the $\gamma$-ray band, thus supporting the above hypothesis. However, thanks to the recently released Pass 8 data from Fermi-LAT, which is more sensitive at lower energies and thus important for high redshift objects, it is now possible to search distant blazars in the $\gamma$-ray band and constrain their SEDs in a far better way than in the past. We search for the detection of significant $\gamma$-ray emission from all four high redshift blazars studied in this work (including J1656-3303 which is included in 3FGL catalog) and report $\mathrm{J} 0225+1846$ as a newly detected $\gamma$-ray emitter at $\sim 13 \sigma$ significance. This is the first report of the discovery of the $\gamma$-ray emission from this object and confirms the prediction of Healey et al. (2008). We also find marginal $\sim 2 \sigma$ evidence for the detection of J1430+4205 at $\gamma$-ray energies. Moreover, we search for temporal flux variations among J0225+1846 and J1656-3303 and find the former to exhibit a low amplitude $\gamma$-ray flare around the beginning of the year 2013 (see Figure 2). This source has also shown hints of spectral curvature in its $\sim 7.5 \mathrm{yr}$ average $\gamma$-ray spectrum. 

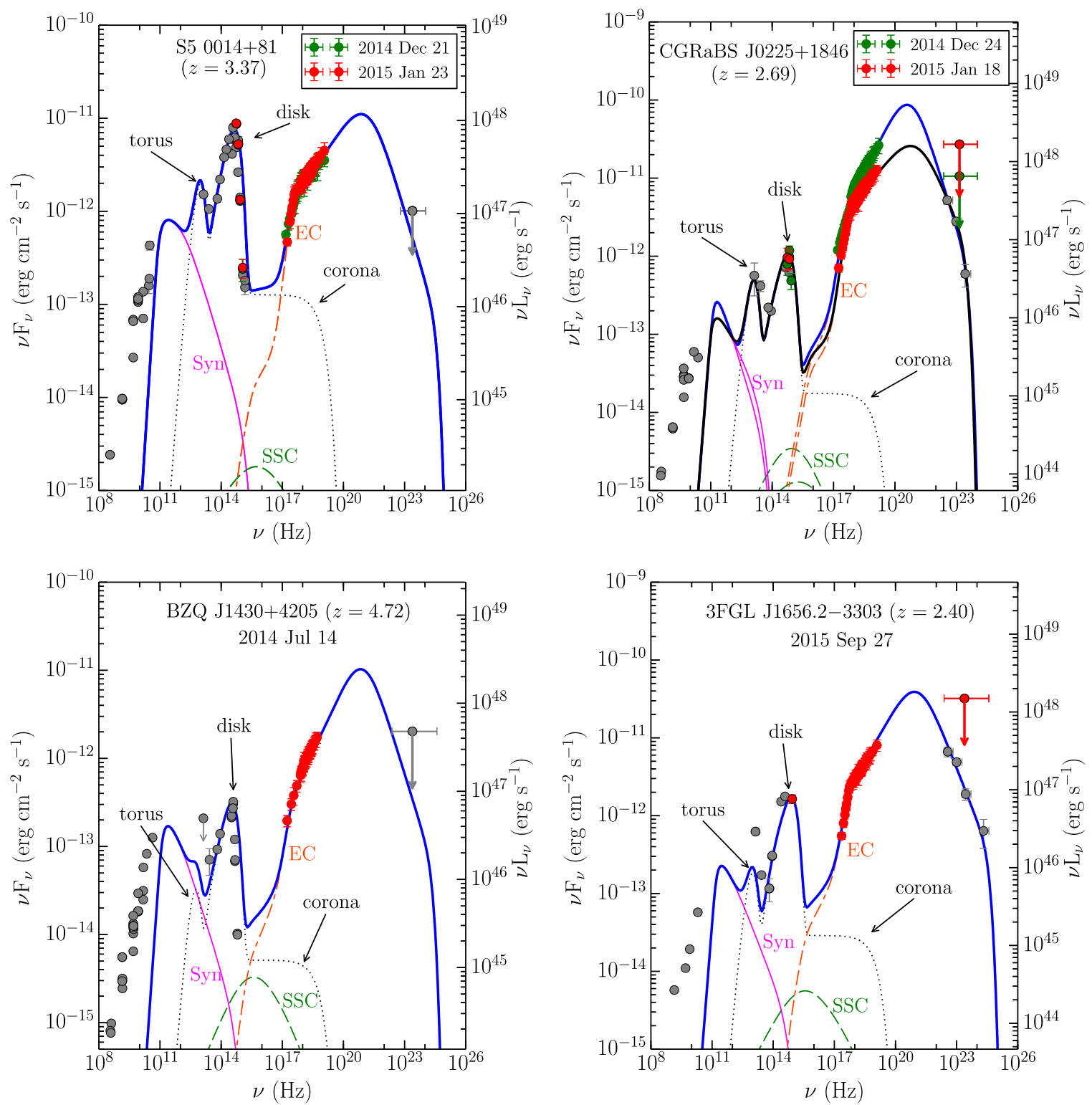

Figure 5. SEDs of high redshift blazars. Contemporaneous data from Swift and NuSTAR are shown by colored circles and non-simultaneous observations are represented by circles. The Fermi-LAT gray circles correspond to the $\sim 7.5 \mathrm{yr}$ average $\gamma$-ray spectrum, and downward arrows are the $2 \sigma$ upper limits. Thermal emission from the torus, the accretion disk, and the X-ray corona is represented by the black dotted line. The pink thin solid, green dashed, and orange dash-dash-dot lines correspond to synchrotron, SSC, and EC emission, respectively. The blue and black thick solid lines are the sum of all the radiative components.

Table 4

List of the Parameters Used in the SED Modeling

\begin{tabular}{|c|c|c|c|c|c|c|c|c|c|c|c|c|c|}
\hline $\begin{array}{l}\text { Name } \\
{[1]}\end{array}$ & $\begin{array}{l}\text { Date } \\
{[2]}\end{array}$ & $\begin{array}{c}M_{\mathrm{BH}} \\
{[3]}\end{array}$ & $\begin{array}{c}L_{\text {disk }} \\
{[4]}\end{array}$ & $\begin{array}{l}n 1 \\
{[5]}\end{array}$ & $\begin{array}{l}n 2 \\
{[6]}\end{array}$ & $\begin{array}{c}B \\
{[7]}\end{array}$ & $\begin{array}{c}U_{\mathrm{e}} \\
{[8]}\end{array}$ & $\begin{array}{c}\Gamma \\
{[9]}\end{array}$ & $\begin{array}{c}\gamma_{\mathrm{b}} \\
{[10]}\end{array}$ & $\begin{array}{l}\gamma_{\max } \\
{[11]}\end{array}$ & $\begin{array}{l}R_{\text {diss }} \\
{[12]}\end{array}$ & $\begin{array}{c}R_{\mathrm{BLR}} \\
{[13]}\end{array}$ & $\begin{array}{l}T_{\text {torus }} \\
{[14]}\end{array}$ \\
\hline $\mathrm{J} 0014+81$ & 2014 Dec 21 & 10.04 & 48.0 & 2.3 & 4.5 & 2.2 & 0.001 & 10 & 116 & 5000 & 0.89 & 1.02 & 500 \\
\hline \multirow[t]{2}{*}{$\mathrm{J} 0225+1846$} & 2014 Dec 24 & 9.40 & 46.9 & 1.9 & 4.7 & 1.0 & 0.023 & 16 & 46 & 1000 & 0.26 & 0.29 & 580 \\
\hline & 2015 Jan 18 & 9.40 & 46.9 & 2.3 & 4.3 & 1.0 & 0.015 & 14 & 71 & 1000 & 0.26 & 0.29 & 580 \\
\hline $\mathrm{J} 1430+4205$ & 2014 Jul 14 & 9.54 & 47.0 & 1.9 & 4.5 & 1.6 & 0.022 & 14 & 79 & 3500 & 0.20 & 0.31 & 400 \\
\hline J1656-3303 & 2015 Sep 27 & 9.30 & 47.0 & 2.3 & 4.4 & 1.0 & 0.051 & 14 & 68 & 3500 & 0.15 & 0.32 & 400 \\
\hline
\end{tabular}

Note. Column [1]: name of the object; Column [2]: date of observation; Column [3]: mass of the central black hole, in log scale; Column [4]: accretion disk luminosity, in erg s ${ }^{-1}$, in log scale; Columns [5] and [6]: spectral indices of the electron energy distribution; Column [7]: magnetic field, in Gauss; Column [8]: particle energy density, in erg cm${ }^{-3}$; Column [9]: bulk Lorentz factor; Column [10]: break Lorentz factor; Column [11]: maximum Lorentz factor; Column [12]: distance of the emission region from central black hole, in parsec; Column [13]: size of BLR, in parsec; and Column [14]: characteristic temperature of the dusty torus, in Kelvin. There are no differences in the SEDs of J0014+81 for the 2014 December 21 and 2015 January 23 observations (see Figure 5). Therefore, here we provide modeling parameters associated with 2014 December 21 SEDs only. 
Table 5

Various Jet Powers (in erg s ${ }^{-1}$ ) Derived from the SED Modeling of the High Redshift Blazars Studied in this Work

\begin{tabular}{lccccc}
\hline \hline $\begin{array}{l}\text { Name } \\
{[1]}\end{array}$ & $\begin{array}{c}\text { Date } \\
{[2]}\end{array}$ & $\begin{array}{c}P_{\text {mag }} \\
{[3]}\end{array}$ & $\begin{array}{c}P_{\text {rad }} \\
{[4]}\end{array}$ & $\begin{array}{c}P_{\text {ele }} \\
{[5]}\end{array}$ & $\begin{array}{c}P_{\text {kin }} \\
{[6]}\end{array}$ \\
\hline J0014+81 & 2014 Dec 21 & 47.13 & 45.92 & 44.72 & 47.35 \\
$\mathrm{~J} 0225+1846$ & 2014 Dec 24 & 45.80 & 46.76 & 45.57 & 48.18 \\
& 2015 Jan 18 & 45.68 & 46.21 & 45.26 & 47.93 \\
$\mathrm{~J} 1430+4205$ & 2014 Jul 14 & 45.86 & 46.30 & 45.20 & 47.70 \\
$\mathrm{~J} 1656-3303$ & 2015 Sep 27 & 45.21 & 46.21 & 45.32 & 47.89 \\
\hline
\end{tabular}

Note. Column [1]: name of the object; Column [2]: date of observation; Columns [3]-[6]: jet powers in the magnetic field, radiation, electrons, and protons, respectively, in log scale.

\subsection{Soft X-Ray Flattening}

A flattening of the soft X-ray spectrum is observed in all four blazars considered in this study. In fact, this feature is observed in several high redshift quasars (e.g., Eitan \& Behar 2013) and, in earlier studies, it was attributed to the presence of absorbing material intrinsic to the source environment (e.g., Fabian et al. 2001a, 2001b). However, the low level of reddening observed at optical-UV energies does not support the above hypothesis. This is because, in some cases, the column densities derived from the X-ray spectral fitting reaches as high as $N_{\mathrm{H}} \approx$ $10^{23} \mathrm{~cm}^{-2}$ (Fabian et al. 2001a) and accordingly the reddening in the optical-UV band would be large $\left(A_{\mathrm{V}} \sim 100\right)$, which is against the observations. An alternative solution, the so-called "warm absorber," was proposed by invoking an extreme gasto-dust ratio, probably because of the high ionization state of the objects (e.g., Fabian et al. 2001a). On the other hand, the recent work by Tavecchio et al. (2007) (see also, Ghisellini et al. 2007; Foschini 2009) suggests that the soft X-ray flattening originates from the jet emission.

Switching M2 to use an ionized absorber (modeled with an XSTAR grid) offers an improved fit to the XRT/NuSTAR spectrum of $\mathrm{J} 0225+1846$, although still not as good as the fit with M3 (the broken power law). Additionally, this model requires an extremely high column density $\left(\sim 5 \times 10^{23} \mathrm{~cm}^{-2}\right)$ to account for the observed curvature in this spectrum. The combination of ionization and high column density predicts very strong iron absorption lines around 6-7 keV in the rest frame, redshifted to just below the NuSTAR band (see Figure 6). The strength of these features is such that they would be easily detected by the more sensitive EPIC-pn spectrum or the highresolution RGS spectrum in the case of $\mathrm{J} 0225+1846$.

More generally, there is a large disagreement between the column densities measured from the broadband XRT/NuSTAR spectra and the XMM-Newton spectra. The column densities from the broadband spectra are consistently several times larger (over 10 times in the case of J1656-3303). If the spectral curvature is caused by absorption, these values should be the same, or at least there should not be a consistent trend if the absorption is variable. However, if the spectral curvature is more gradual and intrinsic to the source, then we would expect exactly this trend, as more curvature is found in the broadband spectra and thus a higher column is needed to fit the data. A similar trend is observed with the broken power-law model (M3), where the break energy is lower in the XMM-Newton spectra and the power-law indices are lower. This is exactly as expected from fitting such a model to a smooth curve.

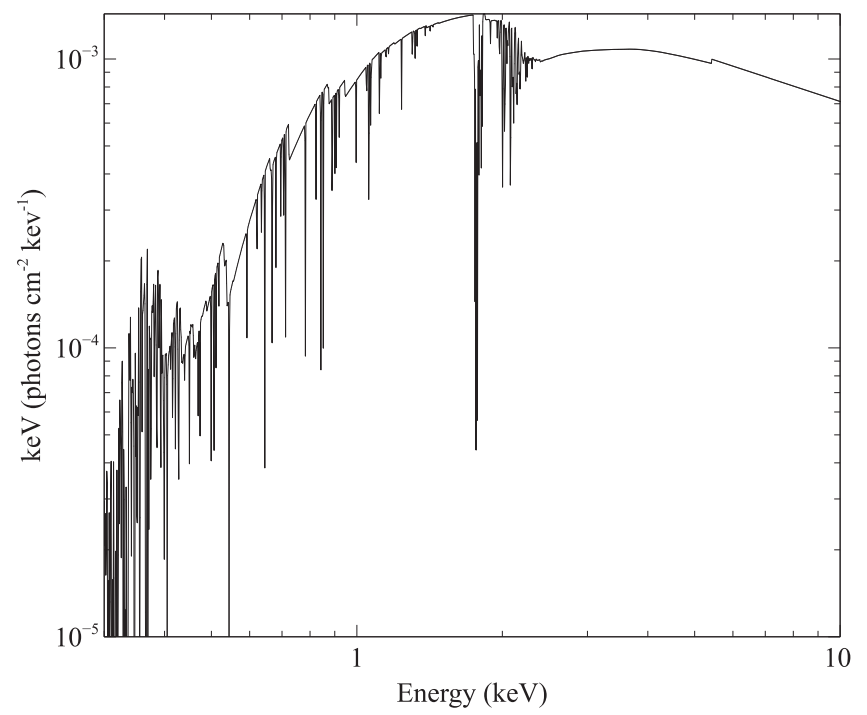

Figure 6. Ionized absorption model for the broadband NuSTAR/Swift spectrum of J0225+1846. The energy scale is in the observer's frame. Strong iron absorption lines are visible, redshifted to around $2 \mathrm{keV}$, which would be easily detectable in the XMM-Newton spectrum.

While it is always possible that the absorbing column to each source has changed between the XMM-Newton and NuSTAR observations, it is extremely unlikely that all four sources would change in the same manner. When combined with the better fits to the broadband spectra found using the broken power-law model, this is very strong evidence that the spectral curvature is not caused by absorption, but is instead an intrinsic part of the blazar X-ray spectrum, thus confirming the results obtained in earlier studies (Ghisellini et al. 2007; Tavecchio et al. 2007; Foschini 2009).

\subsection{Jet Emission and the Soft X-Ray Deficit}

The broadband SEDs of all four blazars can be well reproduced using a simple one-zone leptonic emission model (Figure 5). Following the blazar sequence, their synchrotron peak lies at sub-millimeter or self-absorbed frequencies and this shifting leaves the accretion disk spectrum visible at opticalUV energies. By modeling the optical-UV spectrum with a standard accretion disk model, we constrained both the black hole masses and the accretion luminosities in all four sources. All the objects are found to host black holes of more than a billion solar mass at their centers, with the central black hole mass of $\mathrm{J} 0014+81$ estimated as $\sim 10$ billion solar mass, thus making it one of the most massive black holes ever found in radio-loud quasars beyond redshift $z=3$. For a consistency check, we compare the black hole masses obtained from the SED modeling approach with those from single epoch optical spectroscopic line information. Varshalovich et al. (1987) reported the following relation using the optical spectroscopy of $\mathrm{J} 0014+81$.

$$
\frac{M_{\mathrm{BH}}}{R_{\mathrm{BLR}}} \approx 1.3 \times 10^{10}\left(\frac{M_{\odot}}{\mathrm{pc}}\right)
$$

Following Ghisellini \& Tavecchio (2009), we find the BLR radius as $\sim 1 \mathrm{pc}$ (see Table 4). This suggests $M_{\mathrm{BH}} \approx$ $1.3 \times 10^{10} M_{\odot}$ which is similar to that obtained from accretion disk modeling. For J1430+4205, we use C IV line and continuum parameters from the Sloan Digital Sky Survey data 
archive and adopt the empirical relations of Shen et al. (2011). This gives the central black hole mass as $\sim 1.7 \times 10^{9} M_{\odot}$ which agrees within a factor of two to that derived by the SED modeling approach. We consider the published $\mathrm{C}$ IV line parameters from Masetti et al. (2008) and the derived black hole mass of $\mathrm{J} 1656-3303$ is $\sim 6.3 \times 10^{8} M_{\odot}$ which reasonably matches within a factor of three to that obtained from modeling. On the other hand, we could not obtain any spectroscopic line information from the literature for J0225 +1846 . Furthermore, the accretion disk is also found to be extremely luminous in all the sources with $L_{\text {disk }}>10^{46} \mathrm{erg} \mathrm{s}^{-1}$. The level of synchrotron emission is constrained from the archival radio data and is kept low enough so as not to overproduce the observed IR-optical-UV SED. Another deciding factor is that we needed enough injected electron power to reproduce the high energy X-ray to $\gamma$-ray SED via IC mechanism. Furthermore, in all the sources, the $0.3-79 \mathrm{keV}$ $\mathrm{X}$-ray spectra are very hard and the associated $\gamma$-ray spectra are steeply falling, as expected from high redshift blazars. We interpret the entire X-ray to $\gamma$-ray SED as a result of the EC mechanism with the BLR as a the primary reservoir of the seed photons. This sets the location of the emission region within the BLR in all four objects, a feature generally seen in many high redshift FSRQs (e.g., Ghisellini et al. 2010). We constrain the spectral indices of the underlying electron population by reproducing the X-ray spectra and also by keeping in consideration the upper limits observed at LAT energies. Moreover, although not used for modeling, the long time average Fermi-LAT spectra assist us in having an idea about the typical shape of the falling part of the EC process.

In our sample of four blazars, two of them, J0014+81 and J0225+1846, were contemporaneously observed by NuSTAR and Swift in 2014 December and 2015 January. Having more than one episodes of monitoring enables us to study the possible variations in their SEDs. As can be seen in Figure 5, the SED of J0014+81 does not show any variation between these two epochs and, in fact, is similar to archival observations. On the other hand, J0225+1846 became fainter in 2015 January compared to 2014 December. It should be noted that the slopes of the falling EC spectrum in both the activities states are primarily decided by the Fermi-LAT upper limits (and with NuSTAR observations which controls the EC peak), but we also keep in mind its typical $\gamma$-ray spectral shape as revealed by the long time average LAT spectrum (Figure 5). An interesting observation is the detection of the low value of the $\gamma$-ray flux upper limit at the time of the high X-ray state (2014 December, see also, Figure 2), which makes the falling EC spectrum steeper (Figure 5). In the 2015 January observation, on the other hand, $\mathrm{J} 0225+1846$ was in a relatively low X-ray state but the obtained upper limit indicates it to be slightly brighter in $\gamma$-rays, thus making the EC spectrum flatter at $\gamma$-ray energies. Now, by comparing these two SEDs and also noting that the $N U S T A R$ spectrum is harder in the brighter state, we find that during the high activity phase, the EC peak shifted to lower frequencies, in accordance with the traditional blazar sequence.

It is interesting to compare the physical properties of these four high redshift blazars with other blazars at similar redshifts and also with comparatively nearby objects. The main motivation here is to search for any possible trend or evolution of the physical parameters, such as the $L_{\text {disk }}$ and total jet power $\left(P_{\text {jet }}\right)$ over cosmic history of time and also to check the relative position of the four sources studied here. With this in mind, we collect $L_{\text {disk }}$ and $P_{\text {jet }}$ values of all the 226 objects studied by Ghisellini et al. (2014), covering up to redshift 3.04. For higher redshift objects, we choose those sources from BZCAT (Massaro et al. 2015) that have $z>3.04$, exhibit a hard $\mathrm{X}$-ray spectrum (X-ray photon index $<1.7$, see, e.g., Saez et al. 2011), and are highly radio-loud ${ }^{6}$ (radio-loudness factor $R>100$, e.g., Kellermann et al. 1989). We then perform the broadband SED modeling of all these objects using the same leptonic emission model. We plot the variation of $L_{\text {disk }}$ and $P_{\text {jet }}$ with respect to the redshift and show them in the top panels of Figure 7. We also present a plot between $L_{\text {disk }}$ and $P_{\text {jet }}$ in the bottom left panel of Figure 7. Moreover, we overplot all the four sources considered in this work (including both the activity states of J0225+1846). We find a positive correlation for $L_{\text {disk }}$ and $P_{\text {jet }}$ with redshift, respectively, and also between the two (Figure 7). This is not unexpected because, at higher redshifts, only the most powerful objects are visible. However, there are a few points worth noticing. First, $L_{\text {disk }}$ increases up to a redshift of $\sim 3-3.5$ and after that it appears to saturate around $10^{47} \mathrm{erg} \mathrm{s}^{-1}$. Second, similar behavior is visible in the $P_{\text {jet }}$ versus redshift plot, where beyond redshift $4 P_{\text {jet }}$ tend to deviate more toward the lower power from the best linear fit. A comparison of the slopes obtained from the best linear fitting of $L_{\text {disk }}$ and $P_{\text {jet }}$ versus redshift indicates that $P_{\text {jet }}$ does not

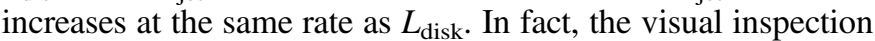
suggests a relative decrease in $P_{\text {jet }}$ for $z>3$ objects. These findings are further confirmed in the $L_{\text {disk }}$ versus $P_{\text {jet }}$ plot, where slope of the obtained linear fit $(0.56 \pm 0.03)$ is steeper than that derived by Ghisellini et al. (2014). As can be seen in Figure 7, the change of slopes has occurred due to high redshift $(z>3)$ blazars occupying a high $L_{\text {disk }}$ and relatively low $P_{\text {jet }}$ regime. In other words, although $P_{\text {jet }}>L_{\text {disk }}$ is true for relatively low redshift blazars (Ghisellini et al. 2014), the same may not hold for blazars beyond redshift 3 or 4 . However, we caution that a strong claim cannot be made for two reasons: (i) blazars beyond redshift 4 are primarily discovered in IR-optical surveys (e.g., Yi et al. 2014) and thus are relatively bright in the optical band, implying a more luminous accretion disk even with moderate power jets, and (ii) it is difficult to precisely measure the jet power in blazars beyond redshift 3 due to a lack of good quality, high energy X-ray and $\gamma$-ray observations. None of the sources, beyond redshift 3.04, are present in the 3FGL catalog and only few of them have sensitive X-ray data from facilities like NuSTAR. This could be the reason for the observed large scatter in $P_{\text {jet }}$ for blazars beyond redshift 3 . Observations from $N u S T A R$ are crucial to confirm/reject the trend seen in Figure 7. On a different note, it is also of great interest to consider another class of AGN with powerful relativistic jets, the radio-loud narrow line Seyfert 1 (RL-NLSy1) galaxies. The detection of significant $\gamma$-ray emission from some of these RL-NLSy1 galaxies haa confirmed the idea that these sources also host powerful relativistic jets similar to blazars (see, e.g., Foschini 2012 for a review). We collect the $L_{\text {disk }}$ and $P_{\text {jet }}$ information for a sample of RL-NLSy1 galaxies studied by Foschini et al. (2015) and plot them along with the known blazars in the bottom right panel of Figure 7. As can be seen, the RL-NLSy1 galaxies do not follow the $L_{\text {disk }}-P_{\text {jet }}$ correlation seen among blazars and

\footnotetext{
6 The selection of the sources from BZCAT is the part of our ongoing investigation of the physical properties of high redshift quasars and the details of the analysis will be presented in a forthcoming publication.
} 

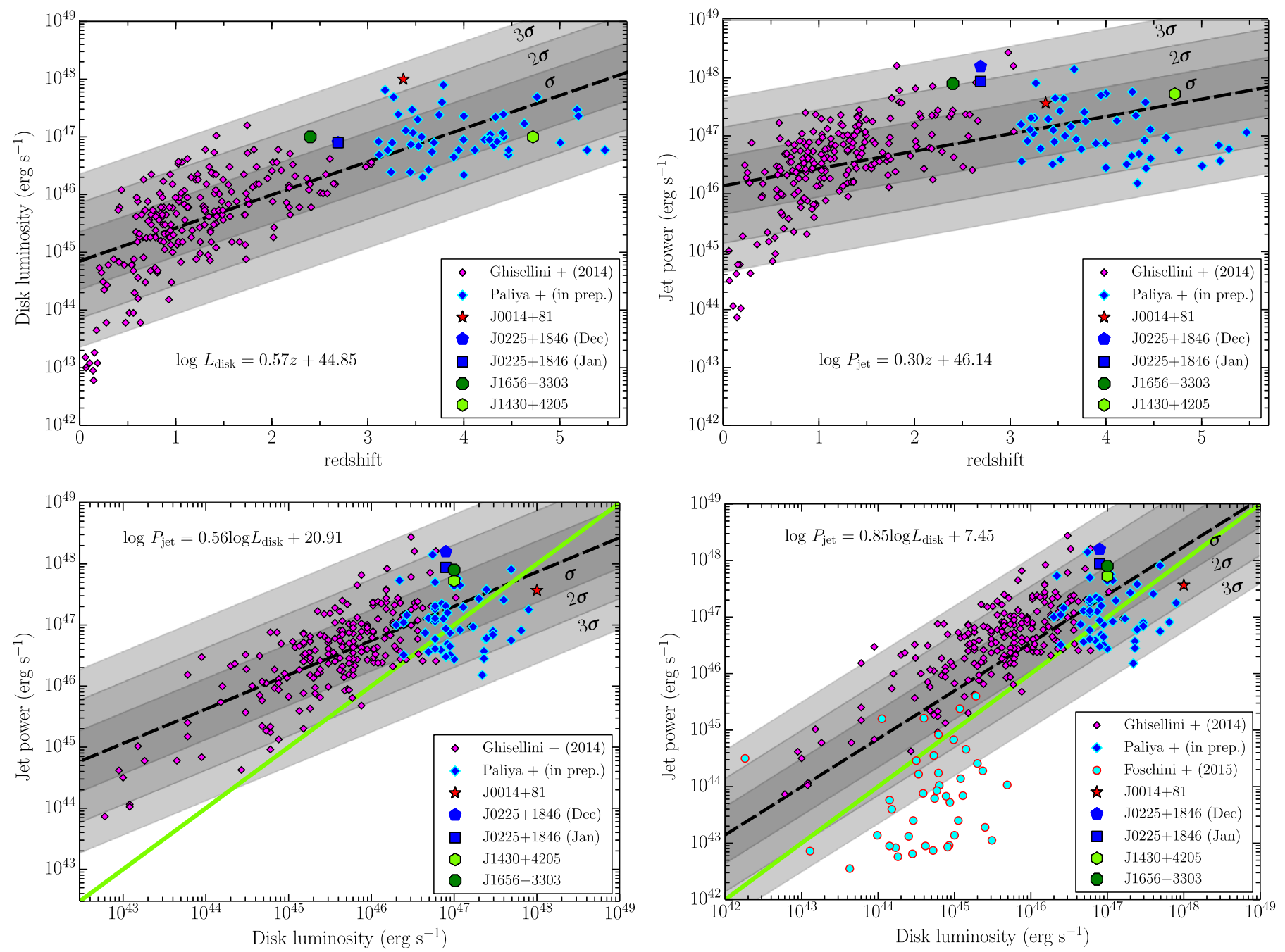

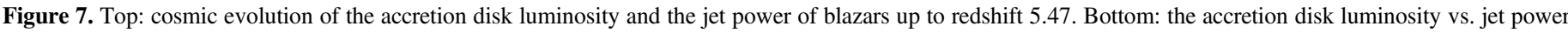

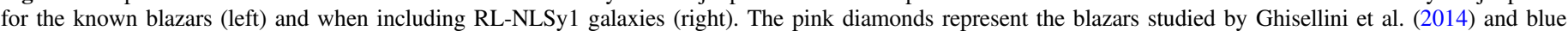

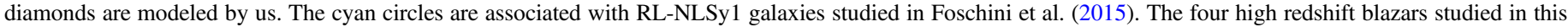

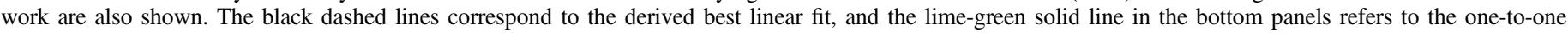
correlation of the plotted quantities. The shaded areas represent $\sigma, 2 \sigma$, and $3 \sigma$ dispersions with $\sigma=0.5$ dex.

occupy a distinct region where there jet power is considerably lesser than blazars and the disk luminosity is comparable to low jet power blazars. These objects are known to have a high accretion rate (e.g., Zhou et al. 2006) but host a relatively low power jet, possibly due to harboring low mass black holes. ${ }^{7}$ Considering the four sources studied here, we find them to occupy a place in $L_{\text {disk }}$ versus $P_{\text {jet }}$ plot where their jet power exceeds the accretion luminosities, except for J0014+81. Furthermore, in the redshift evolution diagram, they appear to follow the common trend with no major exception.

The soft X-ray deficit observed in many jetted sources can also be reproduced by the intrinsic curvature of the EC emission from the jet (see, e.g., Ghisellini et al. 2007; Sambruna et al. 2007; Tavecchio et al. 2007; Tavecchio \& Ghisellini 2008; Foschini 2009). In fact, the SED model used to reproduce the broadband spectrum of a high redshift blazar does predict a smooth flattening of the X-ray spectrum below few $\mathrm{keV}$, although the accurate measurement of the shape and

\footnotetext{
7 However, once normalized by the black holes mass, the jet powers of RLNLSy1 galaxies are consistent with blazars, indicating the scalability of the jet (Foschini et al. 2015).
}

the location of the break depends on the SED parameters and also on the ambient photon distribution (e.g., Tavecchio et al. 2007). Below the break, the EC slope reflects the shape of the seed photon distribution $\left(F(\nu) \propto \nu^{2}\right.$ for a blackbody distribution). For greater emphasis, we plot an absorbed powerlaw spectrum with redshifted column density $N_{\mathrm{H}}=$ $1.5 \times 10^{23} \mathrm{~cm}^{-2}$ and an EC spectrum, for a source located at a redshift of 2.4 and moving with $\Gamma=20$, in Figure 8 . As discussed above, a break in the EC spectrum is visible at a frequency of $\nu_{\text {break }} \simeq \nu_{\text {seed }} \Gamma^{2} \gamma_{\text {min }}^{2} /(1+z)$, where $\nu_{\text {seed }}$ is the peak frequency of the ambient photon field providing seed photons for IC scattering. Assuming the BLR photon field as a predominant source of seed photons $\left(\nu_{\text {seed }}=\nu_{\text {Ly } \alpha}\right)$ and fixing the minimum energy of the underlying electron population to $\gamma_{\min }=1$, we find that the break frequency depends only on the bulk Lorentz factor. The shapes of both spectra are quite similar down to $\sim 1-2 \mathrm{keV}$ (which will be lower for higher redshift objects), as can be seen in Figure 8. Below this energy, EC asymptotically follows a spectrum $\propto \nu^{2}$ (reflecting the slope of the ambient photons), while the intrinsically absorbed power law drops exponentially. Now, to compare these theoretical 

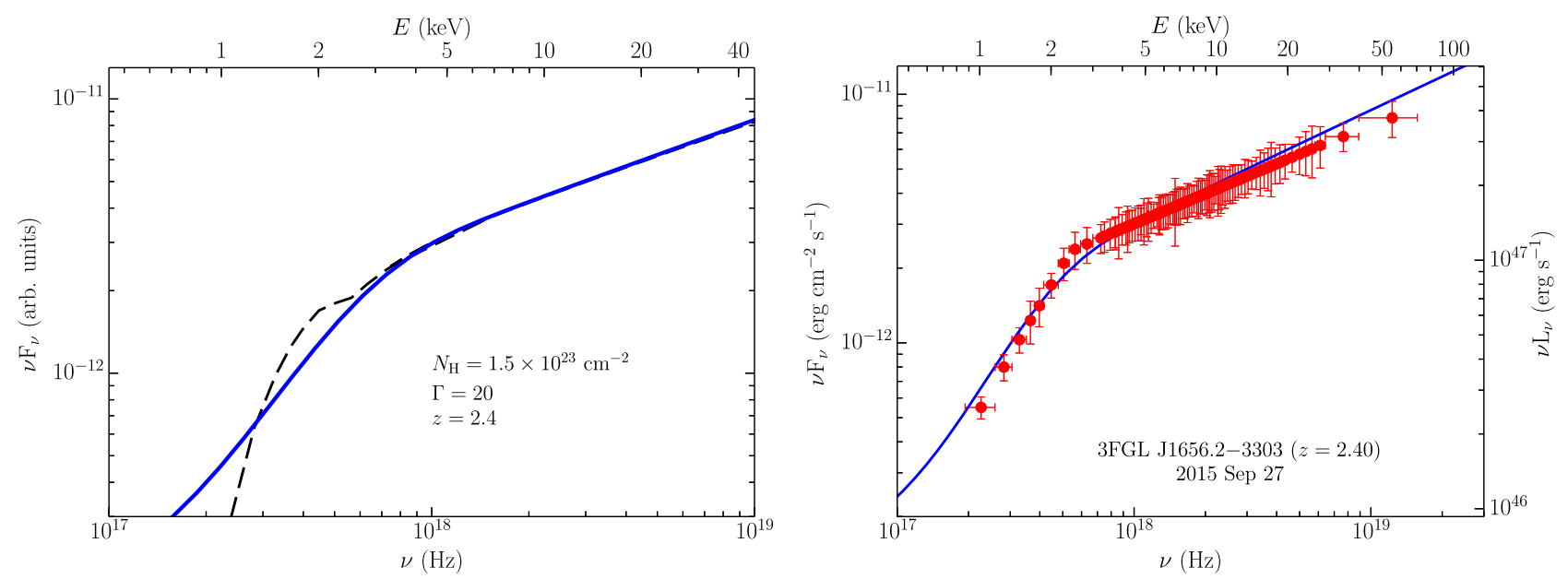

Figure 8. Left: comparison of the EC spectrum (blue solid line) with a power-law (photon index 1.6) spectrum with absorption intrinsic to the quasar frame (black dashed line). The bulk Lorentz factor is assumed to be $\Gamma=20$ to match the absorbed power-law spectrum and, also, we adopt $\gamma_{\min }=1$. The spectra have been arbitrarily normalized. Right: the zoomed version of the X-ray SED of J1656-3303.

arguments with the observations, we plot the zoomed version of the X-ray part of the modeled SED of J1656-3303 in Figure 8. Both the models, discussed above, can reasonably fit the data. However, based on our study (see Section 5.2), the hypothesis of host galaxy/warm absorption can be rejected. This leaves the presence of intrinsic curvature in the jet emission as the most plausible explanation for the soft X-ray deficit in the high redshift blazars and confirms the results of earlier studies (e.g., Ghisellini et al. 2007; Tavecchio et al. 2007; Foschini 2009).

\section{SUMMARY}

In this paper, we study the broadband physical properties of four high redshift blazars, namely J0014+81, J0225+1846, $\mathrm{J} 1430+4205$, and $\mathrm{J} 1656-3303$. We summarize our findings below.

1. A statistically significant $\gamma$-ray emission has been detected from $\mathrm{J} 0225+1846$, confirming earlier predictions of this object as a $\gamma$-ray emitter.

2. The broadband SEDs of all four blazars are typical of their high redshift counterparts, with optical-UV emission dominated by the accretion disk radiation and high energy X-ray to $\gamma$-ray spectra dominated by jet emission processes.

3. All the sources are found to host blacks holes of more than a billion solar mass at their centers and their accretion disk luminosity exceeds $10^{46} \mathrm{erg} \mathrm{s}^{-1}$. Moreover, they occupy the high end of the $L_{\text {disk }}-P_{\text {jet }}$ correlation.

4. A detailed investigation of the joint XRT/ NuSTAR spectral fitting (and also RGS spectral fitting of J0225+1846) favors the jet based origin of the observed soft X-ray flattening in high redshift blazars rather than due to external effects, such as host galaxy absorption and/or a warm absorber.

5. Overall, the blazars beyond redshift 3 tend to deviate from the known one-to-one accretion-jet correlation, however, further observations from facilities, e.g., NUSTAR, are necessary to confirm/reject this hypothesis.
We are grateful to the referee for constructive comments on the manuscript. A.C.F. thanks Greg Madejski for discussions on the curvature of blazar X-ray spectra and acknowledges support from ERC Advanced Grant 340442. This research has made use of data, software, and/or web tools obtained from NASAs High Energy Astrophysics Science Archive Research Center (HEASARC), a service of Goddard Space Flight Center and the Smithsonian Astrophysical Observatory. Part of this work is based on archival data, software, or online services provided by the ASI Science Data Center (ASDC). This research has made use of the XRT Data Analysis Software (XRTDAS) developed under the responsibility of the ASDC, Italy. This research has also made use of the NuSTAR Data Analysis Software (NuSTARDAS) jointly developed by the ASI Science Data Center (ASDC, Italy) and the California Institute of Technology (Caltech, USA). This research has made use of the NASA/IPAC Extragalactic Database (NED) which is operated by the Jet Propulsion Laboratory, California Institute of Technology, under contract with the National Aeronautics and Space Administration. Funding for SDSS-III has been provided by the Alfred P. Sloan Foundation, the Participating Institutions, the National Science Foundation, and the U.S. Department of Energy Office of Science. The SDSSIII web site is http://www.sdss3.org/.

SDSS-III is managed by the Astrophysical Research Consortium for the Participating Institutions of the SDSS-III Collaboration including the University of Arizona, the Brazilian Participation Group, Brookhaven National Laboratory, Carnegie Mellon University, University of Florida, the French Participation Group, the German Participation Group, Harvard University, the Instituto de Astrofisica de Canarias, the Michigan State/Notre Dame/JINA Participation Group, Johns Hopkins University, Lawrence Berkeley National Laboratory, Max Planck Institute for Astrophysics, Max Planck Institute for Extraterrestrial Physics, New Mexico State University, New York University, Ohio State University, Pennsylvania State University, University of Portsmouth, Princeton University, the Spanish Participation Group, University of Tokyo, University of Utah, Vanderbilt University, University of Virginia, University of Washington, and Yale University. 


\section{REFERENCES}

Acero, F., Ackermann, M., Ajello, M., et al. 2015, ApJS, 218, 23

Ackermann, M., Ajello, M., Atwood, W. B., et al. 2015, ApJ, 810, 14

Arnaud, K. A. 1996, in ASP Conf. Ser. 101, Astronomical Data Analysis Software and Systems V, ed. G. H. Jacoby, \& J. Barnes (San Francisco, CA: ASP), 17

Atwood, W., Albert, A., Baldini, L., et al. 2013, arXiv:1303.3514

Baumgartner, W. H., Tueller, J., Markwardt, C. B., et al. 2013, ApJS, 207, 19

Behar, E., Dado, S., Dar, A., \& Laor, A. 2011, ApJ, 734, 26

Breeveld, A. A., Landsman, W., Holland, S. T., et al. 2011, in AIP Conf. Ser. 1358, Gamma Ray Bursts, ed. J. E. McEnery, J. L. Racusin, \& N. Gehrels (Melville, NY: AIP), 373

Burrows, D. N., Hill, J. E., Nousek, J. A., et al. 2005, SSRv, 120, 165

Celotti, A., \& Ghisellini, G. 2008, MNRAS, 385, 283

Condon, J. J., Cotton, W. D., Greisen, E. W., et al. 1998, AJ, 115, 1693

Eitan, A., \& Behar, E. 2013, ApJ, 774, 29

Fabian, A. C., Brandt, W. N., McMahon, R. G., \& Hook, I. M. 1997, MNRAS, 291, L5

Fabian, A. C., Celotti, A., Iwasawa, K., \& Ghisellini, G. 2001a, MNRAS, 324,628

Fabian, A. C., Celotti, A., Iwasawa, K., et al. 2001b, MNRAS, 323, 373

Fabian, A. C., Celotti, A., Pooley, G., et al. 1999, MNRAS, 308, L6

Fabian, A. C., Iwasawa, K., McMahon, R. G., et al. 1998, MNRAS, 295, L25

Foschini, L. 2009, AdSpR, 43, 1036

Foschini, L. 2012, in Proc. Nuclei of Seyfert Galaxies and QSOs, Central Engine \& Conditions of Star Formation, 10

Foschini, L., Berton, M., Caccianiga, A., et al. 2015, A\&A, 575, A13

Fossati, G., Maraschi, L., Celotti, A., Comastri, A., \& Ghisellini, G. 1998, MNRAS, 299, 433

Frank, J., King, A., \& Raine, D. J. 2002, in Accretion Power in Astrophysics, ed. J. Frank, A. King, \& D. Raine (Cambridge: Cambridge Univ. Press), 398

Gehrels, N., Chincarini, G., Giommi, P., et al. 2004, ApJ, 611, 1005

Ghisellini, G., Celotti, A., Fossati, G., Maraschi, L., \& Comastri, A. 1998, MNRAS, 301, 451

Ghisellini, G., Della Ceca, R., Volonteri, M., et al. 2010, MNRAS, 405, 387

Ghisellini, G., Foschini, L., Tavecchio, F., \& Pian, E. 2007, MNRAS, 382, L82

Ghisellini, G., Foschini, L., Volonteri, M., et al. 2009, MNRAS, 399, L24

Ghisellini, G., \& Tavecchio, F. 2009, MNRAS, 397, 985

Ghisellini, G., Tavecchio, F., Maraschi, L., Celotti, A., \& Sbarrato, T. 2014, Natur, 515, 376

Harrison, F. A., Craig, W. W., Christensen, F. E., et al. 2013, ApJ, 770, 103

Healey, S. E., Romani, R. W., Cotter, G., et al. 2008, ApJS, 175, 97

Helmboldt, J. F., Taylor, G. B., Tremblay, S., et al. 2007, ApJ, 658, 203
Hook, I. M., \& McMahon, R. G. 1998, MNRAS, 294, L7

Jansen, F., Lumb, D., Altieri, B., et al. 2001, A\&A, 365, L1

Kellermann, K. I., Sramek, R., Schmidt, M., Shaffer, D. B., \& Green, R. 1989, AJ, 98, 1195

Kuehr, H., Pauliny-Toth, I. I. K., Witzel, A., \& Schmidt, J. 1981, AJ, 86, 854

Kuhr, H., Liebert, J. W., Strittmatter, P. A., Schmidt, G. D., \& Mackay, C. 1983, ApJL, 275, L33

Lawrence, C. R., Bennett, C. L., Garcia-Barreto, J. A., Greenfield, P. E., \& Burke, B. F. 1983, ApJS, 51, 67

Masetti, N., Mason, E., Landi, R., et al. 2008, A\&A, 480, 715

Massaro, E., Maselli, A., Leto, C., et al. 2015, Ap\&SS, 357, 75

Mattox, J. R., Bertsch, D. L., Chiang, J., et al. 1996, ApJ, 461, 396

Okajima, T., Tueller, J., Markwardt, C., et al. 2006, ATel, 799, 1

Page, K. L., Reeves, J. N., O'Brien, P. T., \& Turner, M. J. L. 2005, MNRAS, 364, 195

Piner, B. G., Pushkarev, A. B., Kovalev, Y. Y., et al. 2012, ApJ, 758, 84

Romani, R. W., Sowards-Emmerd, D., Greenhill, L., \& Michelson, P. 2004, ApJL, 610, L9

Roming, P. W. A., Kennedy, T. E., Mason, K. O., et al. 2005, SSRv, 120, 95

Saez, C., Brandt, W. N., Shemmer, O., et al. 2011, ApJ, 738, 53

Sambruna, R. M., Tavecchio, F., Ghisellini, G., et al. 2007, ApJ, 669, 884

Sbarrato, T., Ghisellini, G., Nardini, M., et al. 2013a, MNRAS, 433, 2182

Sbarrato, T., Ghisellini, G., Tagliaferri, G., et al. 2015, arXiv:1510.08849

Sbarrato, T., Tagliaferri, G., Ghisellini, G., et al. 2013b, ApJ, 777, 147

Schlafly, E. F., \& Finkbeiner, D. P. 2011, ApJ, 737, 103

Schwope, A., Hasinger, G., Lehmann, I., et al. 2000, AN, 321, 1

Shakura, N. I., \& Sunyaev, R. A. 1973, A\&A, 24, 337

Shen, Y., Richards, G. T., Strauss, M. A., et al. 2011, ApJS, 194, 45

Tavecchio, F., \& Ghisellini, G. 2008, MNRAS, 386, 945

Tavecchio, F., Maraschi, L., Ghisellini, G., et al. 2007, ApJ, 665, 980

Tueller, J., Markwardt, C., Ajello, M., et al. 2006, ATel, 835, 1

Varshalovich, D. A., Levshakov, S. A., Nazarov, E. A., Spiridonova, O. I., \& Fomenko, A. F. 1987, SvA, 31, 136

Verner, D. A., Ferland, G. J., Korista, K. T., \& Yakovlev, D. G. 1996, ApJ, 465,487

Willingale, R., Starling, R. L. C., Beardmore, A. P., Tanvir, N. R., \& O'Brien, P. T. 2013, MNRAS, 431, 394

Wills, B. J., Wills, D., \& Breger, M. 2011, ApJS, 194, 19

Wilms, J., Allen, A., \& McCray, R. 2000, ApJ, 542, 914

Worsley, M. A., Fabian, A. C., Celotti, A., \& Iwasawa, K. 2004a, MNRAS, 350, L67

Worsley, M. A., Fabian, A. C., Turner, A. K., Celotti, A., \& Iwasawa, K. 2004b, MNRAS, 350, 207

Yi, W.-M., Wang, F., Wu, X.-B., et al. 2014, ApJL, 795, L29

Zhou, H., Wang, T., Yuan, W., et al. 2006, ApJS, 166, 128 